\title{
Lane-Changing Trajectory Prediction Modeling Using Neural Networks
}

\author{
Hamidreza Hamedi and Rouzbeh Shad \\ Department of Civil Engineering Faculty of Engineering, Ferdowsi University of Mashhad, Mashhad, Iran \\ Correspondence should be addressed to Rouzbeh Shad; r.shad@um.ac.ir
}

Received 26 October 2021; Revised 16 January 2022; Accepted 22 January 2022; Published 17 February 2022

Academic Editor: Ozgur Baskan

Copyright (c) 2022 Hamidreza Hamedi and Rouzbeh Shad. This is an open access article distributed under the Creative Commons Attribution License, which permits unrestricted use, distribution, and reproduction in any medium, provided the original work is properly cited.

\begin{abstract}
Concerning autonomous driving, lane-changing (LC) is essential, particularly within complicated dynamic settings. It is a challenging task to model LC since driving behavior is complicated and uncertain. The present study adopts a dual-layer feedforward backpropagation neural network involving sigmoid hidden neurons and linear output neurons for evaluating intrinsic LC complexity. Furthermore, the estimation and validation of the model were performed by large-scale trajectory data. Empirical LC data were obtained from the Next Generation Simulation (NGSIM) project for training and testing the neural network-based LC model. The findings revealed that the introduced model could make precise LC predictions of vehicles under small trajectory errors and satisfactory accuracy. The present work assessed LC beginning/endpoints and velocity estimates by analyzing the vehicles around. It was observed that the neural network model yielded almost the same predictions as the observational LC trajectories as well as following vehicle trajectories on the original and target lanes. Furthermore, for LC behavior characteristic validation, the neural network-produced LC gap distributions underwent comparisons to real-life data, demonstrating the characteristics of LC gap distributions not to differ from the real-life LC behavior substantially. Eventually, the introduced neural network-based LC model was compared to a support vector regression-based LC model. It was found that the trajectory predictions of both models were adequately consistent with the observational data and could capture both lateral and longitudinal vehicle movements. In turn, this demonstrates that the neural network and support vector regression models had satisfactory performance. Also, the proposed models were evaluated using new inputs such as speed, gap, and position of the subject vehicle. The analysis findings indicated that the performance of the proposed NN and SVR models was higher than the model with new inputs.
\end{abstract}

\section{Introduction}

It is expected that an interconnected environment contributes to the solving of a large number of transportation problems in association with mobility, efficiency, environmental impacts, and safety. Connected environments help drivers navigate the current and future driving conditions, particularly information on likely but unobserved hazards. Consequently, drivers can make lane-changing (LC) decisions at higher safety and information levels within connected environments [1]. Carelessly performed LC could be a hazardous maneuver. LC is a complicated driving process, for which it is required to match the speed in the present lane with speed in the target lane and identify a proper gap within the greatest lane to ensure the $\mathrm{LC}$ intention recognition of the driver by the other drivers and good LC [2]. The LC and car-following (CF) models in traffic flow theory perform lateral and longitudinal interaction analyses while driving before the detailed description of LC and CF behavior. The CF model has been long and extensively studied. In comparison to the CF state, the pressure and workload of drivers are substantially enhanced in an LC process, exposing the driver to a high level of hazard and error [3-6]. Several studies presented empirical evidence proposing LC and CF behaviors to be majorly responsible for oscillation formation and enhancement on freeways with multiple lanes [7-9]. Typically, traveling speed heterogeneity between various lanes triggers the LC maneuvers of vehicles. LC behavior is 
involved in traffic congestion. LC occurring at the onset of congestion might reduce the traffic capacity on account of undesirable LC behavior, inducing and propagating stop-go shockwaves. Earlier works demonstrated LC to induce frequent changes in the speeds and gaps on the original and target lanes. A complete lane-changing decision (CLACD) modeling framework that explains both the mandatory and discretionary lane-changing behaviors is developed. An integrated approach is employed to model discretionary lane-changing behavior by combining the target lane selection using the utility theory approach and the gap acceptance behavior using a game theory approach. Results reveal that the CLACD models can effectively capture the observed DLC decisions with high accuracy. Furthermore, the CLACD model shows a realistic prediction of traffic flow patterns compared with the utility theory model [10]. This would add to the likelihood of collisions [11-14], disturbance in traffic flow $[15,16]$, and reduced capacity [17-19]. Compared to CF behavior investigation, the study of LC behavior requires a larger amount of data for support. Considering such negative LC effects on the safety and flow of traffic, it remains an interesting context to model LC behavior. LC modeling has recently been of higher interest to researchers since the fabrication of connected automated vehicles (CAVs) enables the easy acquisition of many datasets through advanced sensing devices. Furthermore, it is possible to transmit regular data (including velocity, position, directly-unobtainable data such as the steering angle, vehicle mass, and acceleration) among vehicles, between vehicles to road identities, and from vehicles to the cloud technology [20]. In general, one can classify LC into LC decisions (LCD) and LC implementation (LCI). In the former, drivers have mental motivation for changing lanes based on the around traffic, while the latter refers to a physical procedure, in which vehicles move from a lane to a target one [21]. It is possible to predict LC, such as any driving behavior predictions, in a classification or regression problem. The former case is aimed at vehicle state discretization to allow quicker real-time vehicle behavior distinguishing, while the latter case seeks to make speed and position predictions for vehicle road movement mapping $[22,23]$. Concerning self-driving vehicles, four phases exist, i.e., (1) environment perception, (2) information processing, (3) the behavior prediction of others in the same environment, and (4) driving decision-making [24]. A driver often needs to respond to a lane-changing request of a lanechanger, which is a function of their personality traits and the current driving conditions. Drivers' responses to lanechanging requests were examined in a connected environment using the CARRS-Q Advanced Driving Simulator. Additionally, drivers' response times are modeled using a random parameter accelerated failure time (AFT) hazardbased duration model. Results revealed that drivers tend to be more cooperative in response to a lane-changing request in the connected environment compared with the baseline condition whereby they tend to accelerate to avoid the lanechanging request [25]. Hence, a large number of works were conducted to focus on the two-dimensional prediction of trajectories for the driving behavior mimicking of humans
[26-30]. LC modeling is among the most prominent areas of transportation-related study. Thus, earlier works developed some LC models in recent decades. These models may be classified into two groups: (1) analytical and (2) data-driven models. There are a relatively small number of data-driven and analytical works. This could be attributed to difficult LCI data collection at a large scale [31-34]. Also, earlier works proved that data-driven models outperformed conventional analytical ones in several characteristics, e.g., trajectory accuracy and traffic flow characteristic replication [35-39]. Such studies adopted the neural network (NN) approach in several variants. Generally, the driving behavior of humans is of high degrees of nonlinearity and complexity. Thus, it cannot be easily modeled using conventional shallow machine learning or mathematical approaches [40]. Despite the introduction of some LC models, numerous questions are yet to be answered to realize LC behavior. For instance, analytical LC models obtain an accuracy of $70-80 \%$ in prediction [41]. Also, a significant degree of inconsistency exists between observations and modeled principles [42]. Indeed, it is expected that models of higher accuracy are developed. A small number of studies with careful examination of LCI can be found. An analytical model cannot easily and accurately consider LC uncertainty and diversity $[32,43,44]$. On the other hand, a data-driven model can solely consider influential factors at a particular time [31]. The historical data shortly before the rapid movement of a driver is an essential component [45]. Data-driven and analytical models mostly take into account LCD or LCI separately. This may not lead to complete LC process reproduction consisting of LCI and LCD and their influence on the traffic behavior.

NNs have enjoyed the highest popularity in data-driven LCI and LCD works. Such popularity arises from (1) their capability of dealing with noisy data estimating unlimited complexity extents under nonlinearity and (2) their need for no simplification or prior awareness of solving the problem, unlike statistical approaches [46]. Hunt and Lyons [47] modeled dual carriageway LCD through the BP-NN approach. Their model estimated LCD on the grounds of the busy traffic in the adjacency. They exploited empirical traffic data and simulation results to demonstrate the competence of their model. Li et al. [48] studied LCD prediction using $\mathrm{NN}$ and BF. They incorporated steering wheel angle, and lane line sensor parameters along with in-vehicle CAN bus acquisition characteristics. Comparison of the results to empirical data revealed an accuracy of up to $91.38 \%$. Ke and Wang [49] introduced an LCD approach for the training and learning of connected automated vehicles (CAVs). Their approach included a microscale cellular automata-based simulation model and a BP-NN model, which could make rapid decisions on whether to perform an LC maneuver or keep the lane. Their approach was found to be efficient in the LCD prediction of CAVs. Compared to data-driven LCD approaches, a small number of works were identified to have conducted NN-based LCI investigations. Ding et al. [31] studied real-time LC trajectory prediction by using a duallayer feed-forward BP-NN approach. The review of earlier works suggested that the BP-NN model was valid for LCD and LCI. 
To deal with the barriers of LC modeling, the present work adopts a dual-layer feed-forward backpropagation NN that contains hidden sigmoid neurons and linear output neurons for evaluating the intrinsic LC complexity. Furthermore, the model is estimated and validated using trajectory data at a large scale. The NN may automatically identify essential characteristics that affect the entire LC process by solely observing position data of four vehicles around the intended vehicle, predicting vehicles' behavior. This study mainly seems to develop a neural network approach for LC prediction based on vehicle position data. Empirical vehicle trajectories of the next generation simulation (NGSIM) project were exploited to derive delicate LC data. The NN model is trained, tested, and compared to the field data.

The remainder of the present article is organized as follows: Section 2 reviews the LC modeling literature; Section 3 addresses the research gap and highlights the contributions of the present work; Section 4 represents the examination sites and processed data; Section 5 introduces an $\mathrm{NN}$ model before introducing the inputs and outputs; Section 6 provides a detailed assessment of the introduced models through empirical data of LC; and Section 7 concludes the study.

\section{Literature Review}

LC has recently been as interesting as CF; however, a small number of studies can be found on LC in the literature [50]. A large rate of advancements was seen in connected, autonomous vehicles $[51,52]$ and enhanced insights into the impacts of LC on traffic operations, e.g., traffic build-up, safety, and emissions [53] necessary to construct models with larger advancements. A review of the available LC approaches suggests that $\mathrm{LC}$ trajectory estimation has a high improvement potential. In general, LC maneuvers are divided into mandatory and discretionary LC. The former refers to LC required to achieve a predefined route (on account of diverging, merging, or a lane drop, for example), while the latter refers to pursuing LC speed advantages or driving comfort. Most of such models focus on LC intention and circumstances. They believed LC behavior to occur when LC conditions were judged based on the LC intention. They majorly focused on three safe distance components, including (1) the distance from the leading vehicle of the target lane, (2) the distance from the following vehicle of the target lane, and (3) the distance from the leading vehicle of the current lane. Mahajan et al. [54] proposed an end-to-end machine learning framework to make LC maneuver predictions using unlabeled data and a small number of characteristics. They employed density-based clusters for the identification of LC and lane-keeping maneuvers. Then, they trained a support vector machine (SVM) for learning clustered label boundaries and the automatic labeling of new raw datasets. Subsequently, they introduced the labeled data to a long short-term memory (LSTM) framework for maneuver category prediction. Xie et al. [55] modeled in datadriven settings by using deep learning techniques. They applied an LSTM NN and a deep belief network (DBN) for
LC modeling by incorporating LCI and LCD. Their datadriven model was found to be capable of accurate vehicle LC prediction. A sensitivity analysis was performed, indicating the relative leading vehicle position on the target lane to be the most prominent LC-related factor. Lee et al. [56] introduced an integrated multilane stochastic continuous carfollowing framework. They exploited deep learning for the likelihood estimation of LC maneuvers. Particularly, they introduced an LC maneuver-derived stochastic volatility within a multilane stochastic optimal velocity model (SOVM). Furthermore, they employed a convolutional NN $(\mathrm{CNN})$ for LC maneuver likelihood estimation in their integrated stochastic continuous car-following framework. The findings revealed that the integrated SOVM yielded almost the same predictions as the LC trajectory observations and the following vehicles' trajectories on both the original and target lanes. Zhang et al. [40] employed deep learning and LSTM NN for the simultaneous modeling of LC and CF behaviors. Also, a hybrid retraining constrained (HRC) technique was introduced for further LSTM optimization. The HRC-LSTM model was observed to be capable of accurate LC and CF behavior estimation at the same time under small longitudinal trajectory errors and significant accuracy of LC prediction in comparison to classical techniques.

A review of data-driven LC researches indicates that NNs have been the most exciting instruments. For instance, Tomar et al. [57] adopted a multilayer perceptron (MLP) and introduced an accurate LC trajectory prediction of discrete paths. The MLP was a simple model with a single input, a single hidden layer, and a single output. It was employed to train, test, and predict vehicle trajectories. A detailed effectiveness discussion of backpropagation (BP) NNs was provided by Ding et al. [31] concerning LC trajectory prediction through vehicle records. The $\mathrm{BP} \mathrm{NN}$ was compared to the Elman network model in terms of the accuracy and training time results. According to the test results, the BP NN was capable of accurately predicting the LC behavior of drivers in traffic flow within urban areas. Also, it was verified that the collected data affected vehicle trajectories. Zheng et al. [46] proposed an NN for the complexity evaluation of LC. They exploited trajectory data at a large scale to estimate and validate their model. Also, they employed a multinomial logit (MNL) model, which was most commonly regarded as an LC framework in earlier studies to make comparisons. The $\mathrm{NN}$ was found to have a prediction accuracy of $94.58 \%$ and $73.33 \%$ for the left and right LC samples, respectively, during model estimation. However, the MNL model can correctly predict solely $13.25 \%$ of the right LC samples and $3.33 \%$ of the left LC samples. Despite the substantial accuracy reduction of the two models in the model's validation, the NN predictions were still satisfactory. Dou et al. [35] employed an NN coupled with an SVM to develop a model for mandatory LC prediction at the lane drops of highways. They achieved an accuracy of $78 \%$ and $94 \%$ for merging and nonmerging behaviors via the positions, vehicle gaps, and speed differences as inputs. However, they provided no explicit discussion of the NN structure. Tang et al. $[58,59]$ introduced 
an LC prediction framework by using an adaptive fuzzy NN to judge LC circumstances and perform steering angle prediction during LC. They incorporated the visual search and vehicle operation behaviors of drivers, driving circumstances, and the motion states of vehicles to build a prediction index system of left LCs. Peng et al. [60] proposed a BP NN for LC behavior prediction. Their model could make accurate LC behavior predictions of drivers at least 1.5 seconds earlier than the LC.

Despite the development of some LCI and LCD-based $\mathrm{NN}$ approaches, a large number of questions remain yet to be answered so that LC behavior could be understood. The closely associated problems include the following:

(i) A small number of works were found in the literature on the detailed investigation of LCI. Concerning analytical frameworks, LC uncertainty and diversity cannot be easily taken into account [44]. Concerning data-driven models, earlier works have included solely impact factors at a given time [31].

(ii) To model LC, typically, vehicle speeds and gaps in two lanes (i.e., the current lane and the target lane) at a given time are incorporated into the simulation, whereas the LCD of a driver would depend on the previous traffic states and driving behavior history.

(iii) Earlier works on LC behavior incorporated a large number of parameters, including the space gap, time gap, speed, and acceleration. However, the vehicle positions in the adjacency of the subject vehicle were exploited as the NN input.

\section{Research Gaps and Contributions}

Earlier NN studies mostly identified temporal information, explored current states, or performed action detection after its onset. However, they did not carry out the prediction of future states. Such studies employed offline data processing to analyze the behavior of drivers on account of limited data. Also, several studies can perform training and make future position predictions of LC vehicles in specific discrete path sections rather than throughout the LC path [31]. A review of LC-based NN model indicates that prediction of subject vehicle position with position inputs has not been evaluated yet. For instance, neural network for prediction of lanechanging trajectory based on the past vehicle data is introduced, and inputs like speed and gap are used for NN model [31]. A backpropagation neural network model was developed to predict lane-changing behavior. Lane changing intent time window is determined via visual characteristics extraction of rear-view mirrors [60]. A neural network model to capture the complexity of lane changing is developed, and large-scale trajectory data are employed for model estimation and validation [46]. The present study primarily seeks to propose an NN model for the bold prediction of complete vehicle trajectories through LC behavior. The Levenberg-Marquardt BP algorithm is employed for the training of the NN. However, in the case of impaired memory, the present study uses scaled conjugate gradient BP.
The novelty of the study contains introduced NN model to predict future position of subject vehicle, and the present work exploits the positions of both the subject and adjacent vehicles as the model input. These inputs would maximize the detection capability flexibility of the target by realizing how autonomous vehicles could be implemented in the future. More importantly, the trajectory history is employed rather than instantaneous information at a given time in the NN-LC model. This study can pioneer comprehensive LC behavior simulation through vehicle positions in the adjacency of the subject vehicle based on an NN. The desired trained network output was selected to be the future LC trajectory. The data of various path sections were mixed in inputs for network training and extending the application range of the model. In particular, the contributions of the present work include the following:

(i) Proposing an NN model for perfectly incorporating the effects of the surrounding vehicles on an LC (subject) vehicle

(ii) Introducing the positions of both the subject and adjacent vehicles as the model input

(iii) The NN model is capable of trajectory prediction of LC vehicles while LC is occurring

(iv) Real-life high-resolution data of vehicle trajectories are employed to calibrate and validate the model

(v) Comparison of the introduced neural networkbased LC model with a support vector regressionbased LC model

(vi) Comparison of proposed models with new input variables

\section{LC Trajectory}

4.1. Dataset. The data of the NGSIM project of FHWA (FHWA, 2008) were exploited in the present work. The NGSIM dataset involves the entire vehicle trajectory dataset of surveillance road sections, i.e., position, acceleration, and speed, at a time interval of 0.1 seconds, along with longitudinal and lateral locations that could be utilized for LC maneuver identification. Three vehicle types, including trucks, cars, and motorcycles, were incorporated. These high-fidelity trajectory data have been extensively utilized in traffic flow research in the recent decade [61-68]. Many LC models have undergone calibration and validation via the NGSIM trajectory dataset $[2,35,55,56,69]$. The present work employed the I-80 and US-101 datasets for the construction of LC prediction models. A schematic of the case study sites is demonstrated in Figure 1. As can be seen, an exciting order was applied to perform the left-to-right numbering of lanes. Each of the sites involves an off-ramp and an on-ramp, with expected significant LC activities. In general, LC maneuvers are divided into mandatory and discretionary LC [70]. The present paper incorporated solely cars and selected LC maneuver cases with no following and leading vehicle LC maneuvers relative to an LC vehicle. Due to the discussion of solely discretionary LC, the present work excluded mandatory LC vehicles with on-ramp freeway 

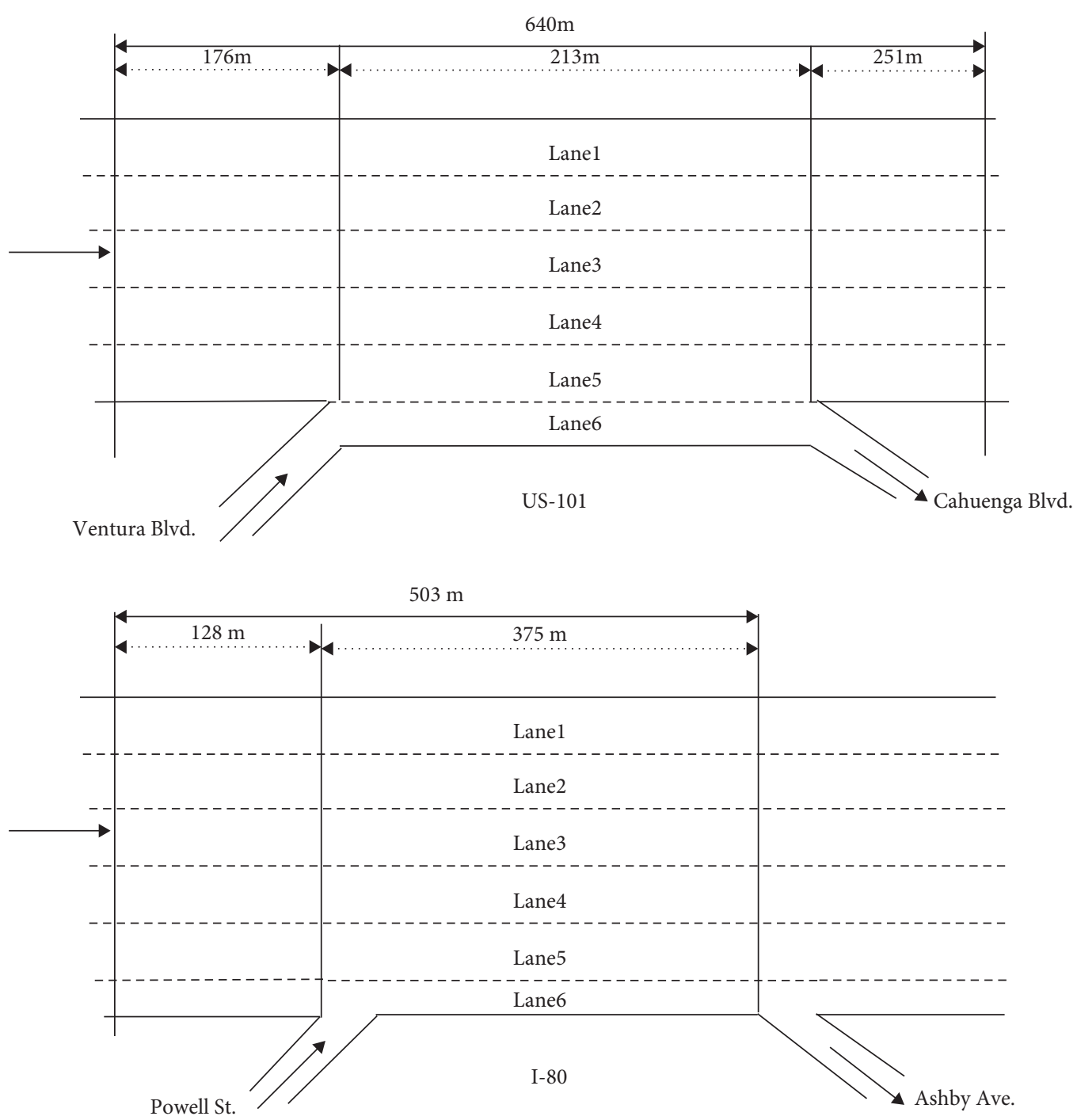

FIgure 1: The case study sites.

entering and off-ramp freeway exiting from the trajectory dataset. Furthermore, this study discarded vehicles with more than LC activities or more than one crosses. Consequently, the trajectories of 2000 vehicles (i.e., 400 complete LC vehicles) were derived. This study defined the duration of LC as the required time for continuous lateral LC movement. A summary of the statistical LC duration results of the vehicles is provided in Table 1 . According to Table 1, the mean LC duration was calculated to be 6.95 seconds. The LC durations of the vehicles were found to be in the range of 1.6-13.8 seconds. Approximately $95 \%$ of the vehicles had an LC duration of shorter than 10 seconds. Hence, the trajectory data of vehicles with an LC duration of below 10 seconds were utilized to eliminate irregular behavior and any noise. Subsequently, the included trajectory dataset involved 100 time-frames (10 s) LC maneuvers on the targeted sections' entire lanes in the I-80 and US-101 freeways.

4.2. LC Prediction Variables. In LC, vehicles perform a twodimensional planar movement instead of a one-dimensional
TABle 1: Statistical LC trajectory results of the NGISM dataset.

\begin{tabular}{lcc}
\hline Duration $(s)$ & Total number of vehicles & Proportion $(\%)$ \\
\hline$<2$ & 5 & 1.25 \\
$2-4$ & 24 & 6 \\
$4-6$ & 88 & 22 \\
$6-8$ & 179 & 44.75 \\
$8-10$ & 79 & 19.75 \\
$10-12$ & 19 & 4.75 \\
$>12$ & 6 & 1.5 \\
Total & 400 & 100 \\
\hline
\end{tabular}

CF movement. LC maneuvers include several vehicle interactions. It is required to carefully determine the variables that could impact the LC decisions of drivers and LC implementation. In general, an LC model incorporates the acceleration and speed data of vehicles on the neighboring lanes and the leading-lagging vehicle gap on the neighboring lanes. These variables pose various impacts on LC behavior modeling [50, 71]. The extraction of information on two- 
dimensional lateral and longitudinal positions of adjacent vehicles for predicting the target vehicle position via an NN is a direct LC behavior modeling technique. Therefore, it is possible to extract further and examine the characteristics of LC behavior. To this end, five vehicles, involving the LC one, that were directly associated with a usual LC process were incorporated, as shown in Figure 2. On the original lane, a vehicle (FC) follows the LC vehicle (SV), and another vehicle (PC) is leading. The same definition was applied to the target lane; a vehicle (FT) follows the LC vehicle, and another vehicle (PT) is leading. According to Figure 2, the beginning of LC behavior is considered to be a position in which the heading of the subject vehicle leaves the present lane direction, while the end of $\mathrm{LC}$ is a position in which the heading of the subject vehicle converges to the target lane direction.

\section{Methodology}

5.1. NN Model. An artificial neural network (ANN) refers to a massive parallel network with simple nonlinear computational components known as neurons. Neurons model several human nervous system functionalities to exploit the human nervous system [72]. ANNs have been often used to approximate nonlinear functions, exhibiting significant benefits for prediction, signal processing, optimization, and pattern identification purposes, in light of their valid and flexible nonlinear self-organization characteristics. ANNs are employed to model a wide range of problems [73-75] and have yielded more significant outcomes than conventional models in several cases. The present work adopted a typical feed-forward BP $\mathrm{NN}$ that had sigmoid hidden neurons along with linear output neurons, as shown in Figure 3.

An $\mathrm{NN}$ is composed of an input layer, one or more hidden layers, and an output layer. The Levenberg-Marquardt backpropagation algorithm was employed for $\mathrm{NN}$ training. However, in the case of impaired memory, scaled conjugate gradient BP would be adopted. Once inputs and the desired output have been introduced to the model, the transferred value propagation is performed from the input layer to the output one through the hidden layers. An NN attempts to learn the relationship between the inputs and output through the free parameters' adaption. A BP NN is mathematically expressed as

$$
Y_{j}=f\left(\sum_{i=1}^{n} w_{j i} x_{i}-b_{j}\right)=f\left(n_{j}\right),
$$

in which column vector $x$ denotes the input vector, row vector $w_{j}$ represents the connection weight vector of neuron $j, b_{j}$ stands for the output threshold, $n_{j}$ is the neuron input, and $f$ is the transfer function.

In order to avoid overtraining and overfitting, various datasets were applied to NN training. It was observed that NN had the smallest error when it was trained by $70 \%$ of the data, as shown in Figure 4.

Generally, a rise in the number of hidden layer neurons improves NN estimation accuracy. However, the increased hidden layer neurons would raise the estimation cost and overfitting issue [76]. To bring a more satisfactory trade-off between accuracy and model cost and overfitting, the mean squared error (MSE) is employed to evaluate NNs with different neuron counts in terms of performance, as shown in Figure 4. The present work utilized the Neural Network Toolbox of MATLAB to construct and implement the NN. During learning, $70 \%$ of the inputs were employed as the training dataset, while the remaining 30\% were exploited as the testing (validation) dataset. This study selected a minimum performance gradient of $10^{-5}$. The NN model was trained and tested using a personal computer with a $1.78 \mathrm{GHz}$ CPU. To obtain optimal performance, NN required a computational time of $76 \mathrm{~s}$. The training and testing of NN lasted for $7584 \mathrm{~s}$. Concerning the hidden layer, the minimum MSE was obtained at a neuron count of eight, as shown in Figure 5. Therefore, the number of hidden layer neurons was selected to be eight in the developed model.

5.2. Inputs and Output. It must incorporate historical motions right before the present as LC is a continuous process while driving. The present study focused solely on the twodimensional prediction of trajectories. Hence, it was required to determine the initial LC states and not distinguishing LC types [77]. Concerning each of the vehicles, the two-dimensional lateral and longitudinal positions of the nearest following and leading vehicles on the present and target lanes were derived through the NGSIM data. The LC model inputs included the time-sequence historical position data of the subject and four adjacent vehicles (Figure 2). Also, the two-dimensional position prediction of the subject vehicle was performed in the following time steps. As mentioned in Subsection 4.1, a total of four hundred complete-LC samples were obtained through the NGSIM data. Two hundred and eighty samples (i.e., 70\% of the dataset) were employed as the training dataset, 60 samples (i.e., $15 \%$ of the data) were used as the validation dataset, and the remaining 60 samples (15\%) were exploited as the testing dataset. Each sample had 10-s LC trajectory data at time intervals of 0.1 seconds. Therefore, each of the samples consisted of a hundred data points. As a result, 28000 and 6000 data points were derived for training and testing, respectively.

Variable $x_{t}$ involves the subject's positions and adjacent vehicles in the detection area, as shown in Figure 2. It is possible to detect the closest leading and following vehicles on the present and target lanes, and the future vector $x_{t}$ in time step $t$ is represented as

$$
x_{t}=\left[P_{s v}^{t}, P_{p c}^{t}, P_{f c}^{t}, P_{p t}^{t}, P_{f t}^{t}\right],
$$

where $P^{t}$ is the vehicle position in time step $t$. It involves local Y (i.e., lateral) and local $X$ (i.e., longitudinal) positions. The positions of $k$ leading time steps are utilized as the inputs of the model. These variables are defined as

$$
\begin{aligned}
& X=\left[x_{t-\Delta t}, x_{t-2 \Delta t}, \ldots, x_{t-k \Delta t}\right], \\
& Y=\left[y_{t-\Delta t}, y_{t-2 \Delta t}, \ldots, y_{t-k \Delta t}\right] .
\end{aligned}
$$




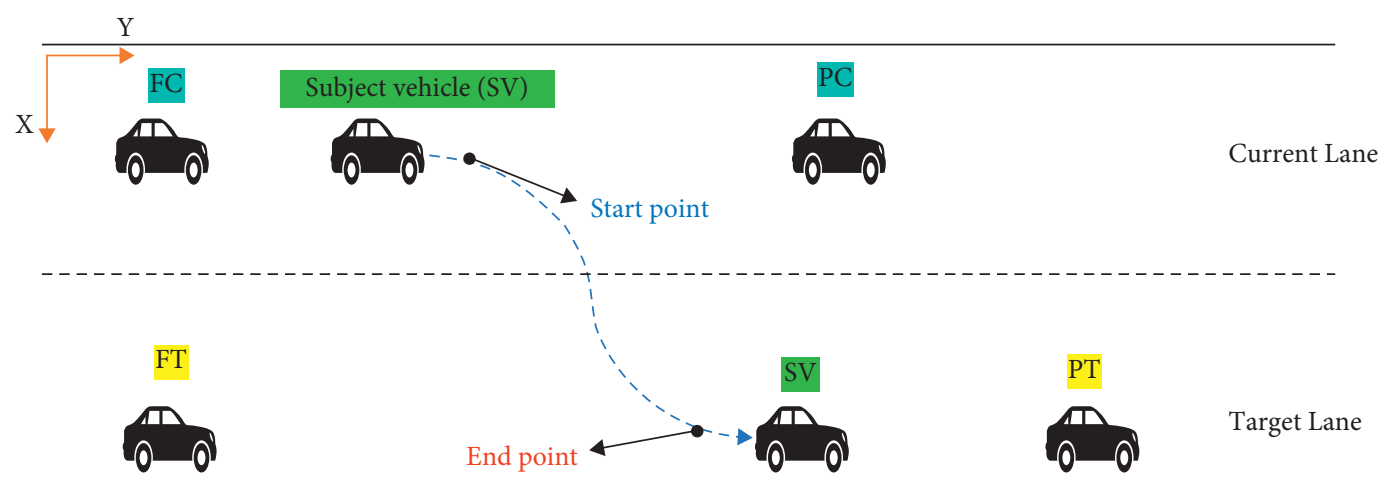

Figure 2: Typical lane-changing process.

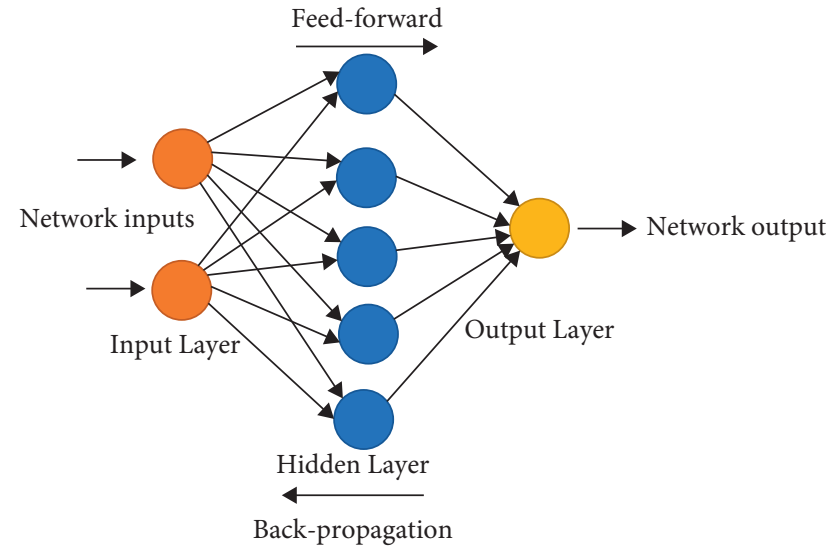

Figure 3: Typical feed-forward back propagation neural network.

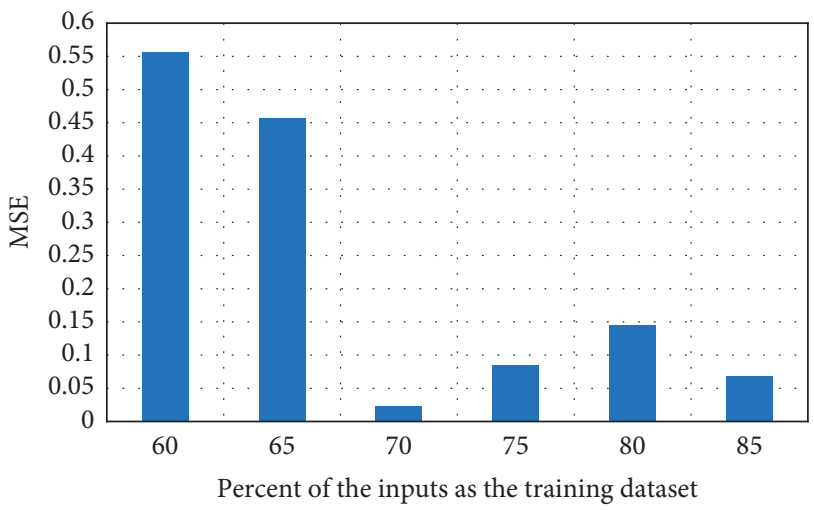

FIgURE 4: Different training datasets for the NN model.

Such inputs can ensure the maximum detection flexibility of the subject vehicle by considering implementation for future autonomous vehicles. The LC outputs include the lateral and longitudinal subject vehicle positions in the next time step $t+\Delta t$ (that is, $\left[y_{t+\Delta t}\right.$ and $\left.x_{t+\Delta t}\right]$ ).

An NN contains a large number of parameters that could impact model performance. Table 2 provides the ultimate parameters.

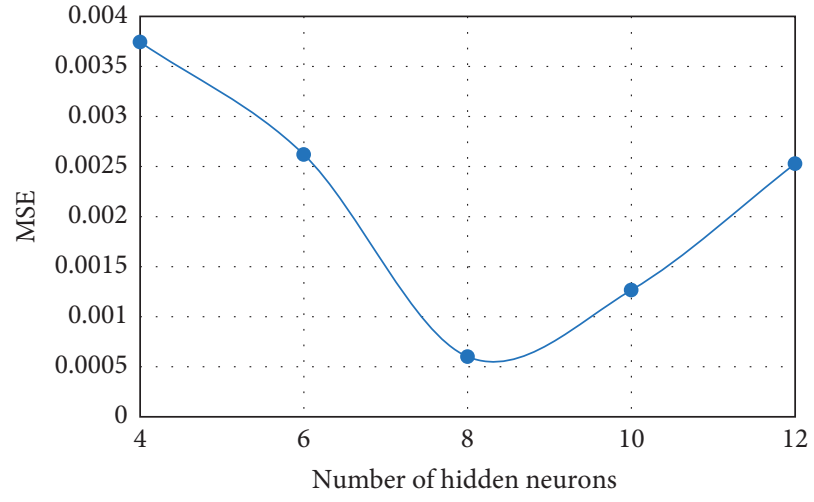

FIGURE 5: Variation of the MSE with the different neuron numbers.

MSE was employed as a performance measure of the NN. It is calculated as

$$
\text { MSE }=\frac{\sum_{i=1}^{T}\left[\left(x_{i}-x_{i}^{\prime}\right)^{2}+\left(y_{i}-y_{i}^{\prime}\right)^{2}\right]}{T} .
$$

T denotes the LC discretization time, $x_{i}$ is the observed longitudinal position, $x_{i}^{\prime}$ is the simulated longitudinal position, $y_{i}$ is the observed lateral position, and $y_{i}^{\prime}$ is the simulated lateral position in time step $k$. The preprocessed data were subjected to normalization before training and testing. The $\mathrm{z}$-score technique was employed as

$$
x^{*}=\frac{x-\mu}{\sigma}
$$

in which $x^{*}$ represents the normalized quantity, $\mu$ is the mean, and $\sigma$ denotes the standard deviation.

5.3. Evaluation Indexes of Model Performance. To model LC behavior, it must perform a separate in-depth analysis of longitudinal and lateral behaviors. Hence, the present work employed several evaluation indexes from three viewpoints. MSE somewhat intuitively judges the prediction accuracy of a model. Concerning the overall accuracy, the model error was employed as an extended longitudinal error variant. It calculates the lateral and longitudinal location and speed errors by using the observational data and simulated results. 
TABLE 2: NN parameters.

\begin{tabular}{lc}
\hline Parameter & Value \\
\hline Input dimension & 10 \\
Output dimension & 2 \\
Historical length $(0.1 \mathrm{~s})$ & 100 \\
Neurons in the hidden layer & 8 \\
Training function & Levenberg-Marquardt BP \\
Adaption learning function & Gradient descent with momentum weight and bias \\
Activation function & Sigmoid \\
Performance function & MSE \\
Transfer function & Hyperbolic tangent sigmoid \\
\hline
\end{tabular}

Concerning longitudinal accuracy, two standard metrics were adopted, including the longitudinal error and mixed gap error. The longitudinal error incorporates both the vertical coordinates and speed. On the other hand, the mixed gap error accounts for the gap errors. The model error, longitudinal error, and mixed gap error are defined as [40]

$$
\begin{aligned}
& \text { model error }=\frac{\sqrt{\sum_{t}\left(x-x^{\prime}\right)^{2}}}{\sqrt{\sum_{t} x^{2}}+\sqrt{\sum_{t} x^{\prime 2}}}+\frac{\sqrt{\sum_{t}\left(y-y^{\prime}\right)^{2}}}{\sqrt{\sum_{t} y^{2}}+\sqrt{\sum_{t} y^{\prime 2}}}+\frac{1}{2} \times \frac{\sqrt{\sum_{t}\left(v-v^{\prime}\right)^{2}}}{\sqrt{\sum_{t} v^{2}}+\sqrt{\sum_{t} v^{2}}}, \\
& \text { long. error }=\frac{\sqrt{\sum_{t}\left(x-x^{\prime}\right)^{2}}}{\sqrt{\sum_{t} x^{2}}+\sqrt{\sum_{t} x^{\prime 2}}}+\frac{1}{2} \times \frac{\sqrt{\sum_{t}\left(v-v^{\prime}\right)^{2}}}{\sqrt{\sum_{t} v^{2}}+\sqrt{\sum_{t} v^{2}}}, \\
& \text { mixed gap error }=\sqrt{\frac{\sum_{t}\left(G-G^{\prime}\right)^{2} /|G|}{\sum_{t} G}},
\end{aligned}
$$

where $x$ is the observed local longitudinal position, $x^{\prime}$ is the simulated local longitudinal position, $y$ is the observed local lateral position, $y^{\prime}$ is the simulated local lateral position, $v$ is the observed speed, $v^{\prime}$ is the simulated speed, $G$ is the observed gap, $G^{\prime}$ is the simulated gap, and $t$ is the frame number ( 0.1 seconds).

\section{Analysis of Results}

6.1. NN-Based LC Model Training. The training convergence rate of $\mathrm{NN}$ is depicted in Figure 6. According to Figure 6, MSE underwent a sharp decline as the iterations increased in number. However, a further rise in the number of iterations did not raise the error when the iterations were adequate. Hence, 100 iterations were applied to the NN training phase, fitting the cross-validation criterion.

6.2. NN-Based LC Model Testing. Table 2 reports the training and testing descriptions of the NN-based LC model for performance evaluation. Figure 7 compares the NN model errors by the training, validation, and testing datasets. A comparison of the training and testing datasets demonstrates the overfitting elimination capability of the model. According to Figure 7, the NN model had rationally satisfactory overall predictive accuracy. For instance, the model error of trajectory predictions varied in the range of 0.02 . Likewise, the longitudinal and mixed gap errors were found to be reasonable. It should be noted that the validation dataset had lower errors than those of the training and testing data in LC prediction, probably due to the smaller LC variance range of the validation dataset than those of the training and testing datasets.

Numerical tests were performed via the NN-based LC model for the trajectory prediction of sixty test-subjected vehicles. MSE was utilized as the index of performance throughout LC. Table 3 shows LC trajectory prediction MSE values of testing data.

Figure 8 depicts the LC trajectory prediction MSE values of twenty vehicles selected randomly (i.e., testing data). The mean MSE value of the tested vehicles was calculated to be nearly 0.0284 . As can be seen, MSE is low, suggesting that the developed LC model is capable of adequately capturing the entire LC process. Figure 9 compares trajectory observations and predictions for the twenty randomly selected vehicles. The results suggest the important consistency of the trajectory predictions and observations. Thus, the model could capture both lateral and longitudinal vehicle motions, and the model is demonstrated to perform properly.

Figure 10 illustrates the LC trajectory predictions and observations of five vehicles. They were selected from the twenty vehicles selected randomly (i.e., testing data). As can be seen, Vehicle 1 had the lowest MSE (Figure 10(a)), whereas Vehicle 155 yielded the highest MSE (Figure 10(c)). The remaining three vehicles were selected randomly. Figures $10(\mathrm{a}), 10(\mathrm{~b})$, and $10(\mathrm{~d})$ depict left LC 


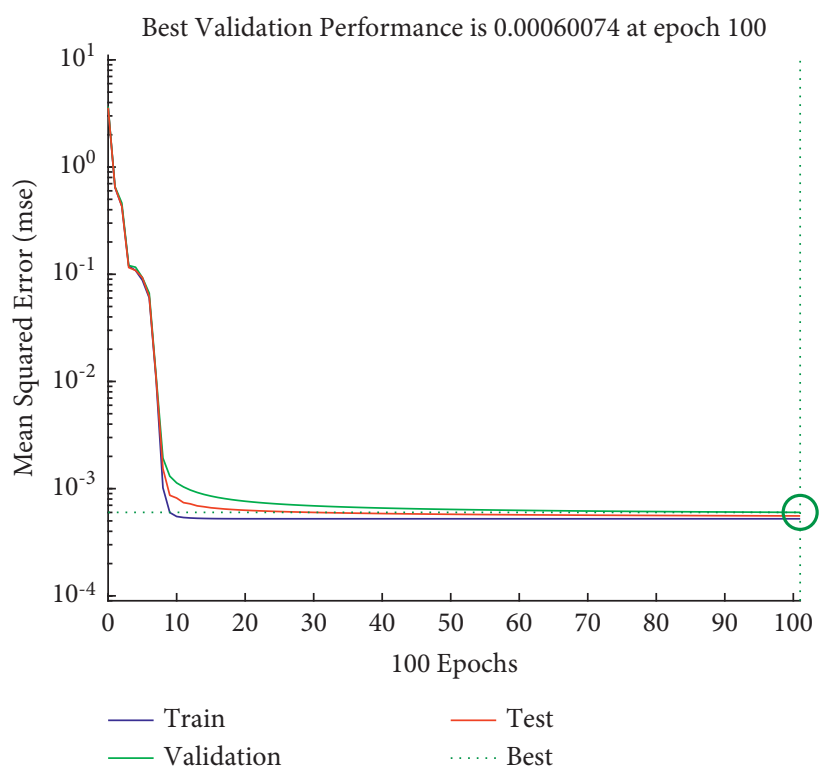

FIGURE 6: The performance curve of the NN model.

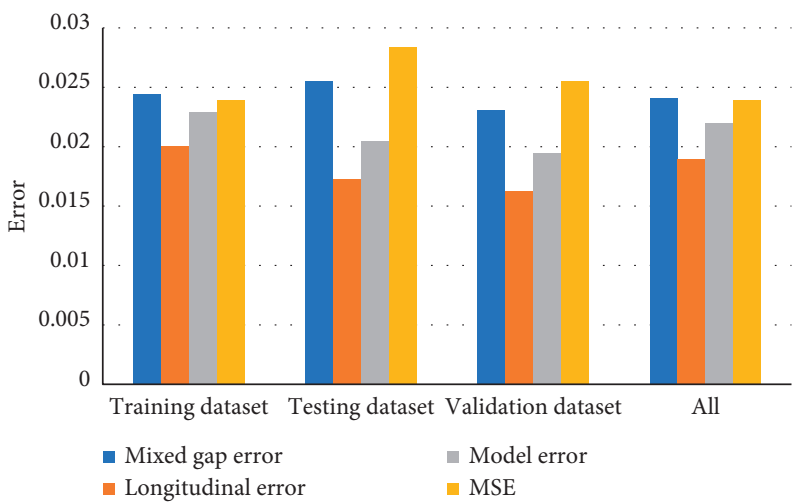

FIgURE 7: The comparison results of different errors.

TABLE 3: LC trajectory prediction MSE values of testing data.

\begin{tabular}{|c|c|c|c|c|c|c|c|}
\hline Vehicle ID & MSE & Vehicle ID & MSE & Vehicle ID & MSE & Vehicle ID & MSE \\
\hline 1 & 0.002856 & 116 & 0.028738 & 227 & 0.038079 & 303 & 0.008232 \\
\hline 5 & 0.038951 & 127 & 0.015417 & 229 & 0.024462 & 305 & 0.010341 \\
\hline 9 & 0.016114 & 132 & 0.032765 & 231 & 0.032455 & 308 & 0.009231 \\
\hline 14 & 0.015715 & 138 & 0.005665 & 235 & 0.013674 & 318 & 0.006383 \\
\hline 17 & 0.016722 & 155 & 0.053034 & 245 & 0.0128 & 332 & 0.009371 \\
\hline 41 & 0.004317 & 169 & 0.005756 & 251 & 0.109571 & 341 & 0.020761 \\
\hline 48 & 0.001693 & 171 & 0.010034 & 261 & 0.032929 & 350 & 0.006996 \\
\hline 67 & 0.016677 & 175 & 0.011424 & 269 & 0.013852 & 353 & 0.073539 \\
\hline 82 & 0.063285 & 191 & 0.021472 & 279 & 0.013526 & 362 & 0.016257 \\
\hline 84 & 0.015718 & 199 & 0.006295 & 283 & 0.026079 & 373 & 0.012606 \\
\hline 89 & 0.016327 & 204 & 0.00577 & 288 & 0.085814 & 378 & 0.019155 \\
\hline 97 & 0.023275 & 206 & 0.010235 & 289 & 0.020792 & 381 & 0.032565 \\
\hline 102 & 0.031238 & 211 & 0.029085 & 293 & 0.351535 & 388 & 0.092071 \\
\hline 111 & 0.007692 & 215 & 0.018323 & 298 & 0.006679 & 393 & 0.011971 \\
\hline 113 & 0.006326 & 218 & 0.00904 & 301 & 0.005626 & 397 & 0.047062 \\
\hline
\end{tabular}




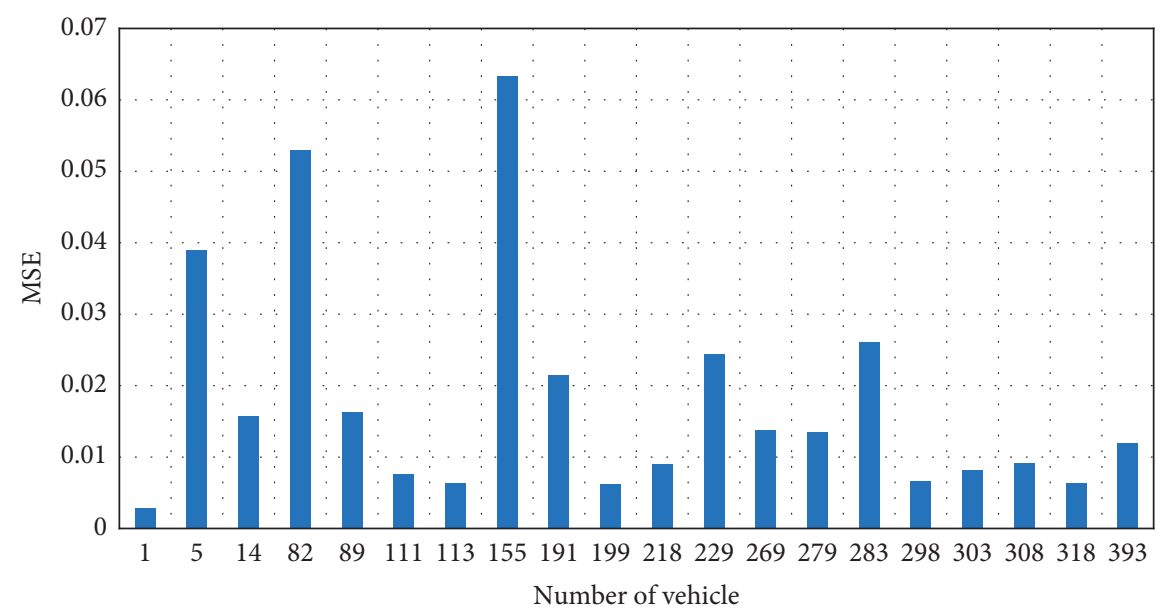

FIGURE 8: LC trajectory prediction MSE values of 20 randomly selected vehicles (testing data).

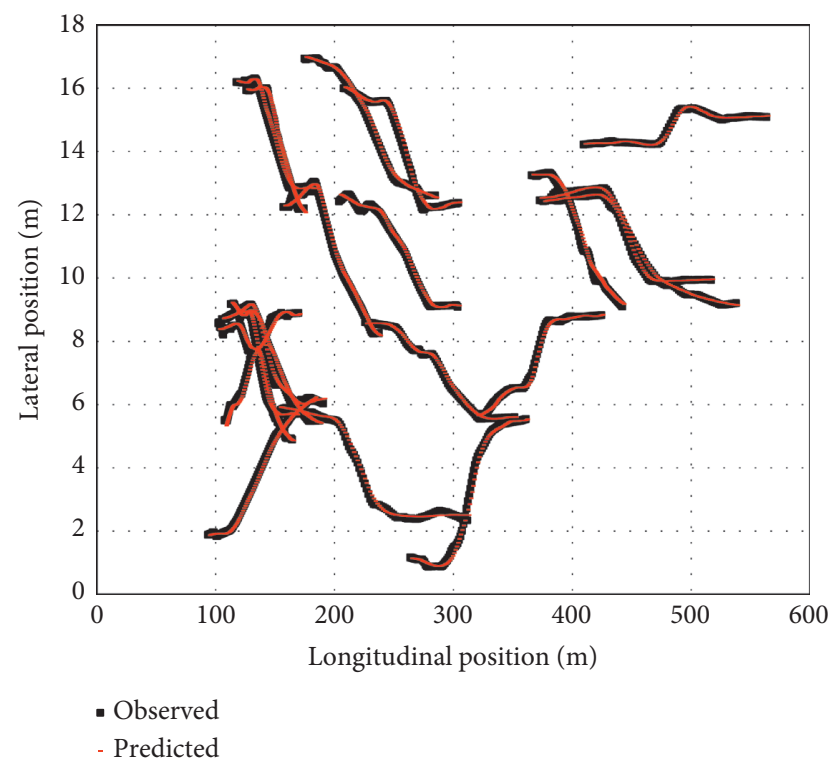

Figure 9: The observed and predicted trajectories of 20 randomly selected vehicles (testing data).

instances, while Figures 10(c) and 10(e) represent right LC instances.

For further performance evaluation of the NN-based LC model, the LC of the vehicles was divided into three LC stages, including

(i) Stage 1: pre-LC: LC preparation before the continuous lateral movement.

(ii) Stage 2: LC: continuous lateral movement.

(iii) Stage 3: adjustment: vehicle speed and direction adjustment of the LC driver after the continuous lateral movement.

Figure 11 depicts MSE values in these stages for the five vehicles. As can be seen, the MSE values of the vehicles were low in Stage 2. This suggests that the developed NN-based LC model is capable of LC trajectory prediction in the LC stage. The errors of prediction, however, are relatively more significant and unstable in the first and third stages-for Vehicle 111 in Stage 1, Vehicle 155 in Stage 3, and Vehicle 298 in Stages 1 and 3. Mainly, Stages 1 and 3 proceed and follow LC implementation, respectively. In these stages, complex impact factors (i.e., the adjacent traffic states) could induce significant driving behavior uncertainty. Also, driving behavior heterogeneity adds to the uncertainty. Therefore, MSE somewhat fluctuates in the first and third stages, as shown in Figure 11. This suggests that a larger number of random impact factors exist in the adjustment stages before LC. As a result, the prediction has a greater difficulty.

According to Figure 2, the beginning of LC behavior is considered to be a position in which the heading of the subject vehicle leaves the present lane direction, while the end of LC is a position in which the heading of the subject vehicle converges to the target lane direction. Thus, Figure 12 and Table 4 show the beginning/end prediction of the five vehicles' testing data. Figure 12 illustrates some LC trajectories (in gray dots) of the testing data. The beginning points are shown in blue, while the endpoints are remarked in red. The beginning/end predictions are close to the observations. This implies that the beginning/endpoint predictions correspond to feasible LC behavior.

6.3. LC Trajectory Predictive Performance. Figure 13 depicts the selected LC driving behavior results of the testing data. The cases with properly estimated LC trajectories and diverse speed variations were selected one by one to fulfill the proposed framework's performance validation for various driving circumstances. Figures 13(a)-13(h) show the speed fluctuations of eight representative LC vehicles for LC maneuvers lasting ten seconds. The black lines represent the average speed prediction of each vehicle, while the red ones stand for the speed observations. These vehicles have no significantly large average speeds since the typical peak hour was utilized for validation. The introduced framework made proper trajectory estimates of all LC vehicles concerning the observed LC speed fluctuations. For the vehicles, the speed 


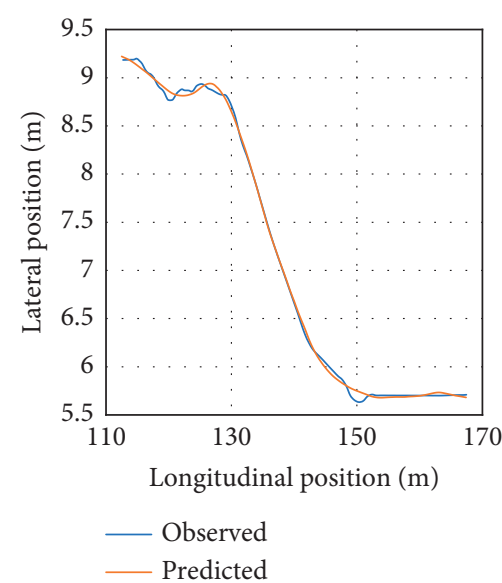

(a)

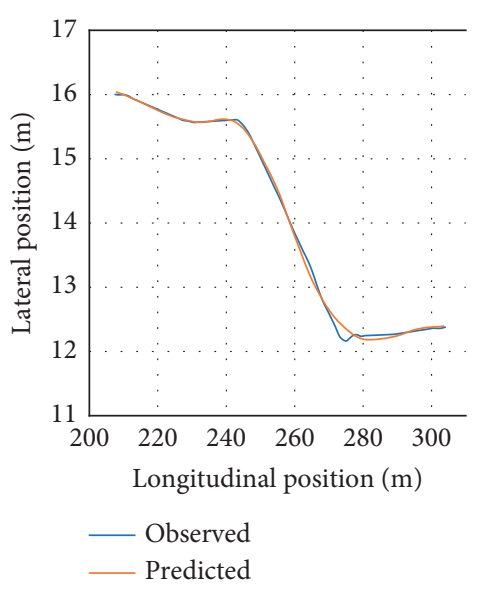

(d)

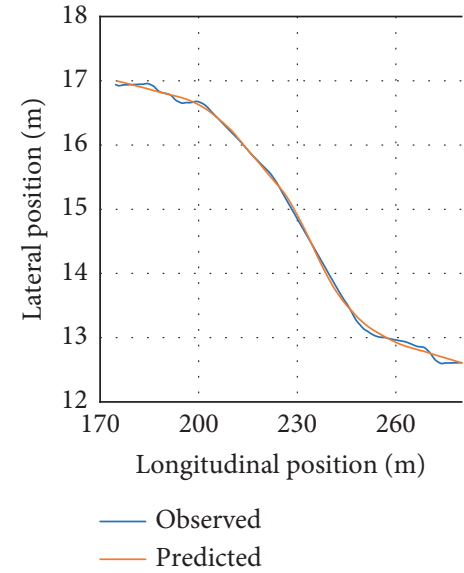

(b)

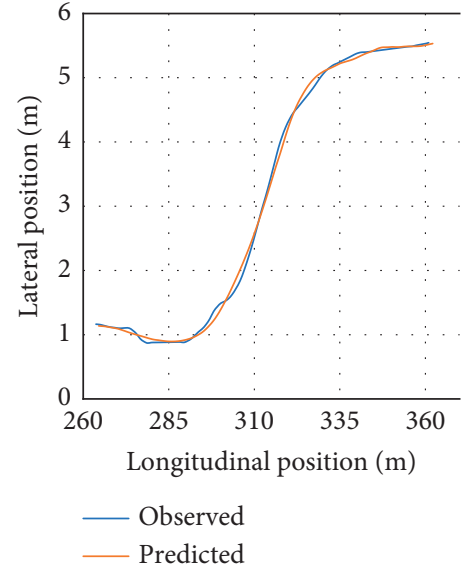

(c)

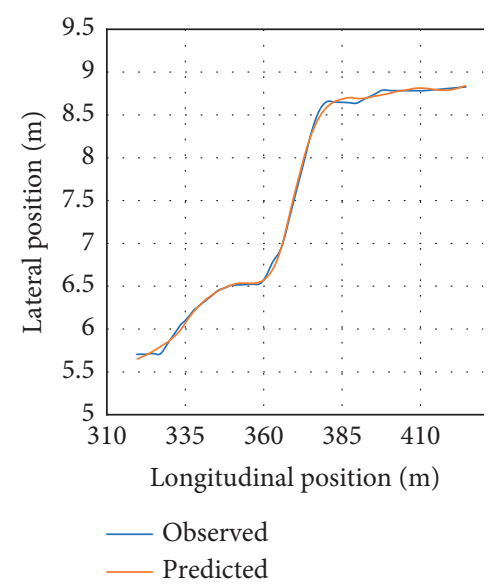

(e)

FIGURE 10: Comparisons of observed and predicted trajectories of vehicles. (a) Vehicle ID: 1. (b) Vehicle ID: 111. (c) Vehicle ID: 155. (d) Vehicle ID: 298. (e) Vehicle ID: 318.

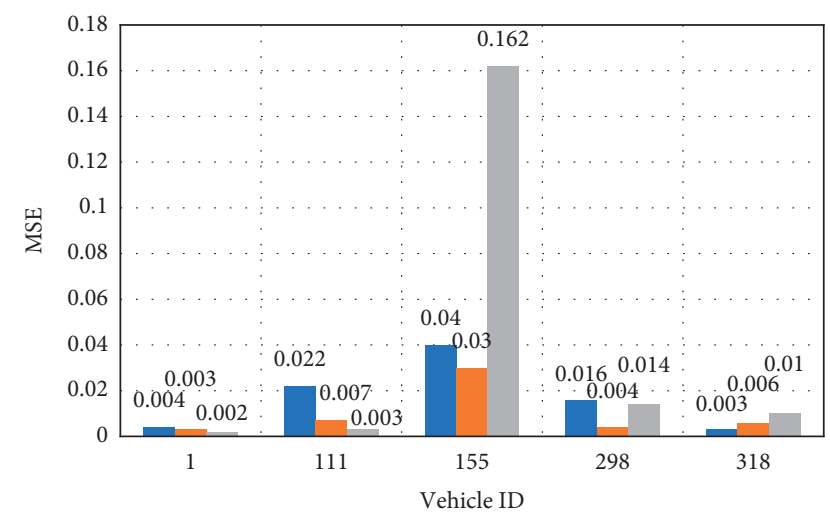

- Stage 1

- Stage 2

- Stage 3

Figure 11: MSE of the predicted LC trajectories in all stages.

prediction MSE was utilized as a performance index throughout the LC process. Vehicles 1 and 298 had the lowest and highest MSE values, as shown in Figures 13(a) and 13(e), respectively. Concerning acceleration, the proposed model exhibited desirable performance in Figures 13(a), 13(c), and 13(f). Also, the LC trajectory estimates were found to be significantly close to the trajectory observations of the vehicles with large speed fluctuations for ten seconds in the LC maneuvers, as shown in Figures 13(b), 13(g), 13(d), and 13(h). For instance, complex impact factors (i.e., the adjacent traffic states) could induce significant driving behavior uncertainty before LC implementation. Also, driving behavior heterogeneity adds to the uncertainty. Consequently, there are large speed fluctuations in Figures 13(g) and 13(d).

Additionally, for the characteristic validation of LC behavior, Figure 14 compares the NN-reproduced LC gap distributions to the real-life data. Also, Welch's $t$-test was employed to evaluate the differences between the NNreproduced distributions and the real-life data. Eventually, the $p$-value was found to be 0.998 . A significantly higher $p$-value than 0.05 suggests a confidence level of $95 \%$ for assuming no significant difference between the prediction and real-life LC results. According to the results, the NN framework is capable of LC prediction and a decent indication of LC characteristics. 

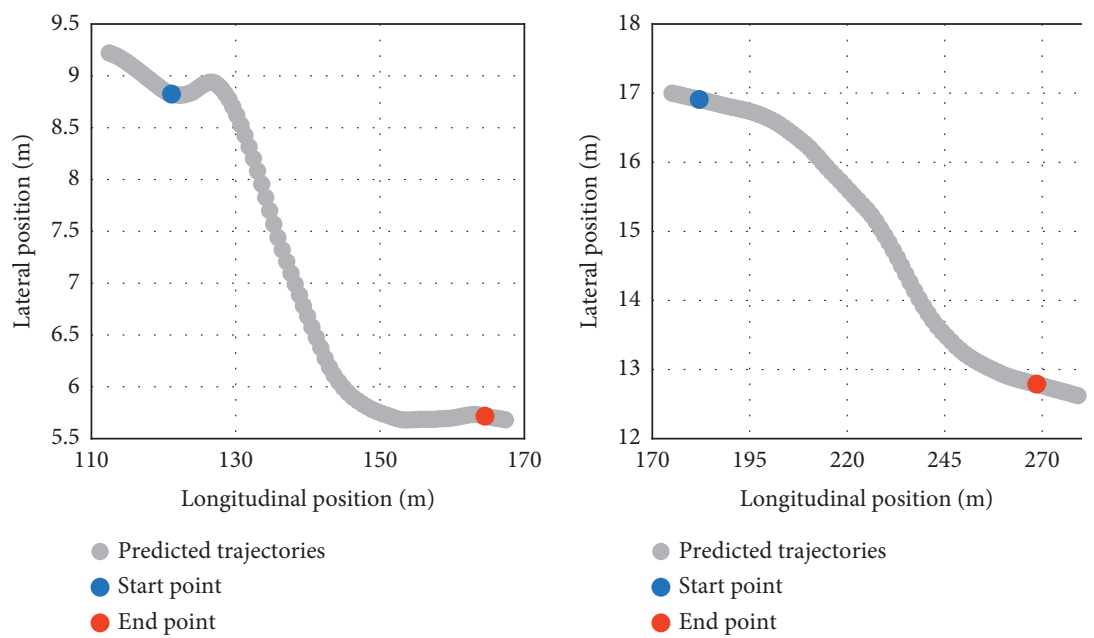

(a)

(b)
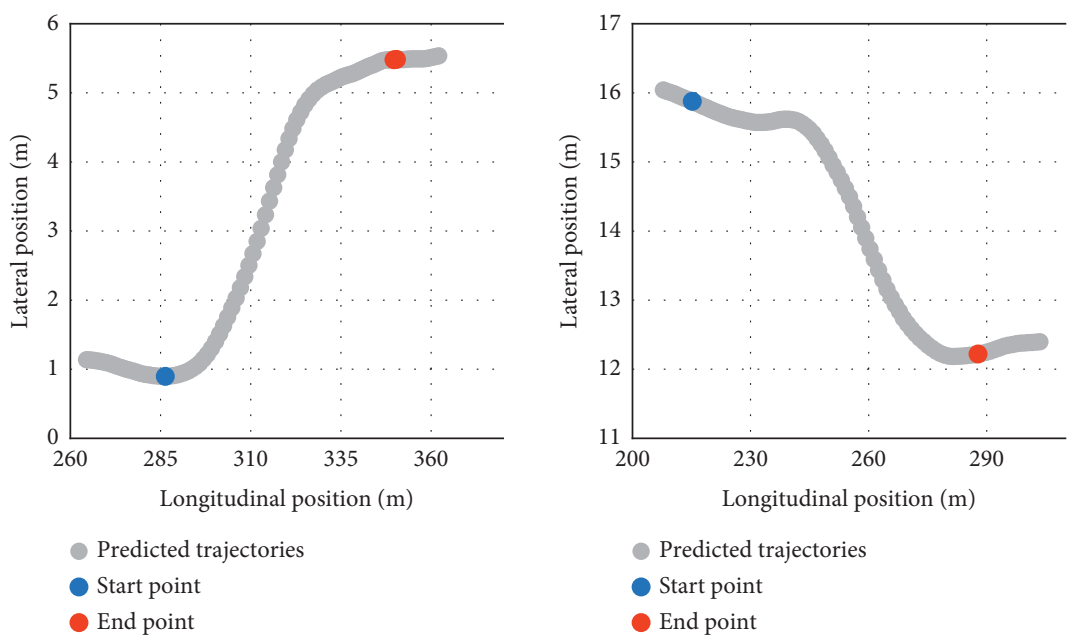

Start point

End point

(d)

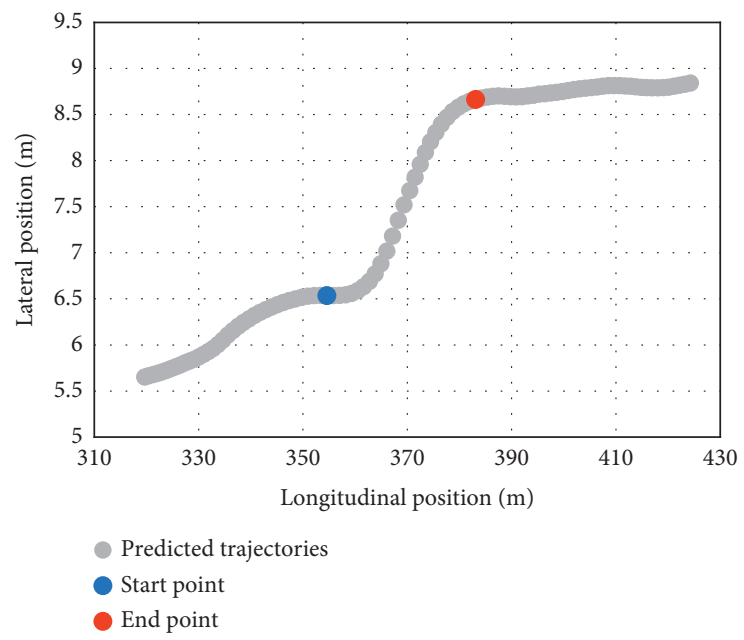

(e)

FIgURE 12: The beginning/endpoint predictions of five vehicles. (a) Vehicle ID: 1. (b) Vehicle ID: 111. (c) Vehicle ID: 155. (d) Vehicle ID: 298. (e) Vehicle ID: 318. 
TABLE 4: Comparison of the beginning/end predictions and observations of five vehicles selected randomly from the testing dataset.

\begin{tabular}{|c|c|c|c|c|c|c|c|c|}
\hline \multicolumn{3}{|c|}{ Beginning point observation } & \multicolumn{2}{|c|}{ Beginning point prediction } & \multicolumn{2}{|c|}{ End point observation } & \multicolumn{2}{|c|}{ End point prediction } \\
\hline $\begin{array}{l}\text { Vehicle } \\
\text { ID }\end{array}$ & $\begin{array}{l}\text { Lateral } \\
\text { position } \\
(\mathrm{m})\end{array}$ & $\begin{array}{l}\text { Longitudinal position } \\
\qquad(\mathrm{m})\end{array}$ & $\begin{array}{l}\text { Lateral } \\
\text { position } \\
(\mathrm{m})\end{array}$ & $\begin{array}{l}\text { Longitudinal position } \\
\qquad(\mathrm{m})\end{array}$ & $\begin{array}{l}\text { Lateral } \\
\text { position } \\
(\mathrm{m})\end{array}$ & $\begin{array}{l}\text { Longitudinal position } \\
\qquad(\mathrm{m})\end{array}$ & $\begin{array}{l}\text { Lateral } \\
\text { position } \\
(\mathrm{m})\end{array}$ & $\begin{array}{l}\text { Longitudinal position } \\
\qquad(\mathrm{m})\end{array}$ \\
\hline 1 & 8.8285 & 121.166 & 8.8231 & 121.114 & 5.70311 & 164.55 & 5.71682 & 164.559 \\
\hline 111 & 16.9454 & 182.208 & 16.9071 & 182.093 & 12.8458 & 268.693 & 12.7861 & 268.629 \\
\hline 155 & 0.88392 & 286.225 & 0.89471 & 286.33 & 5.44982 & 349.878 & 5.47811 & 349.71 \\
\hline 298 & 15.8755 & 215.317 & 15.8763 & 215.227 & 12.2636 & 287.894 & 12.2182 & 287.848 \\
\hline 318 & 6.51967 & 354.62 & 6.53608 & 354.638 & 8.64931 & 383.141 & 8.66336 & 383.134 \\
\hline
\end{tabular}

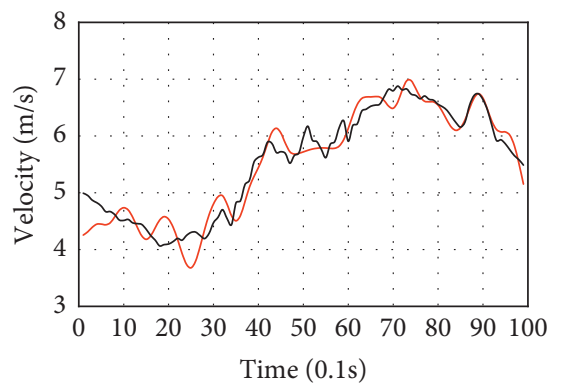

- Observed

Predicted

(a)

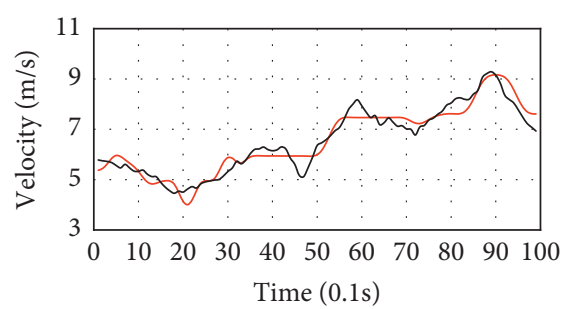

_ Observed

— Predicted

(c)

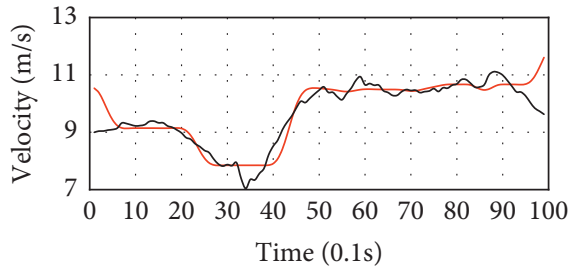

- Observed

- Predicted

(e)

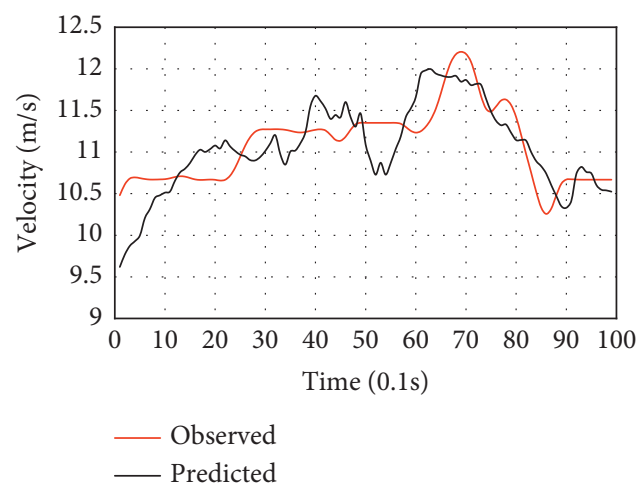

(b)

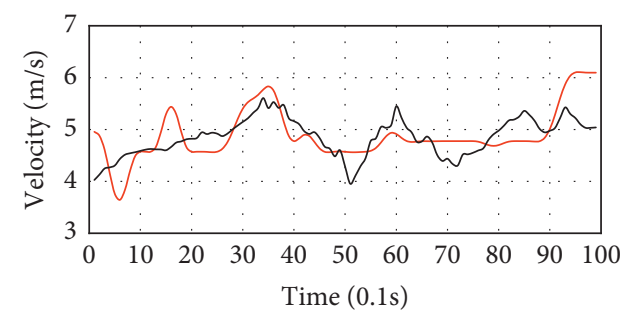

- Observed

- Predicted

(d)

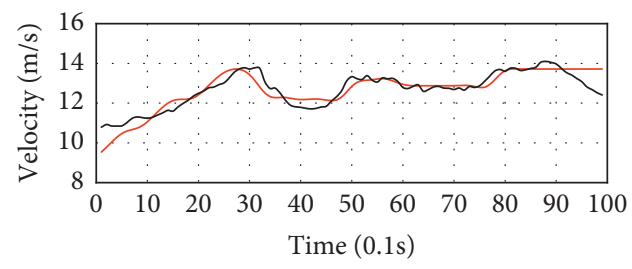

- Observed

— Predicted

(f)

FIGURE 13: Continued. 


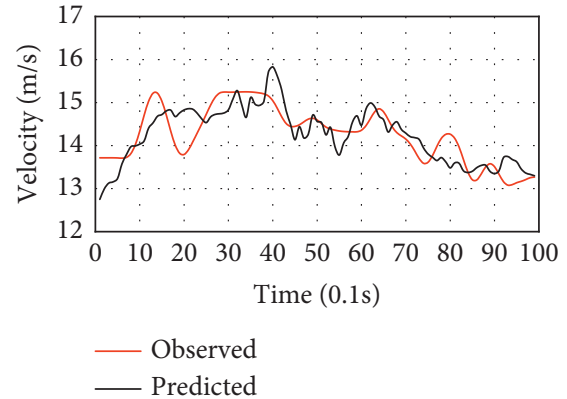

(g)

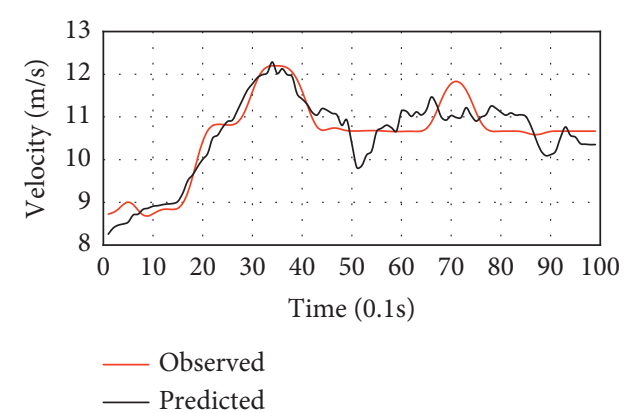

(h)

FIgURE 13: The velocity results of the NN model for lane-changers. (a) Vehicle ID: 1. (b) Vehicle ID: 111. (c) Vehicle ID: 199. (d) Vehicle ID: 218. (e) Vehicle ID: 298. (f) Vehicle ID: 303. (g) Vehicle ID: 308. (h) Vehicle ID: 318.

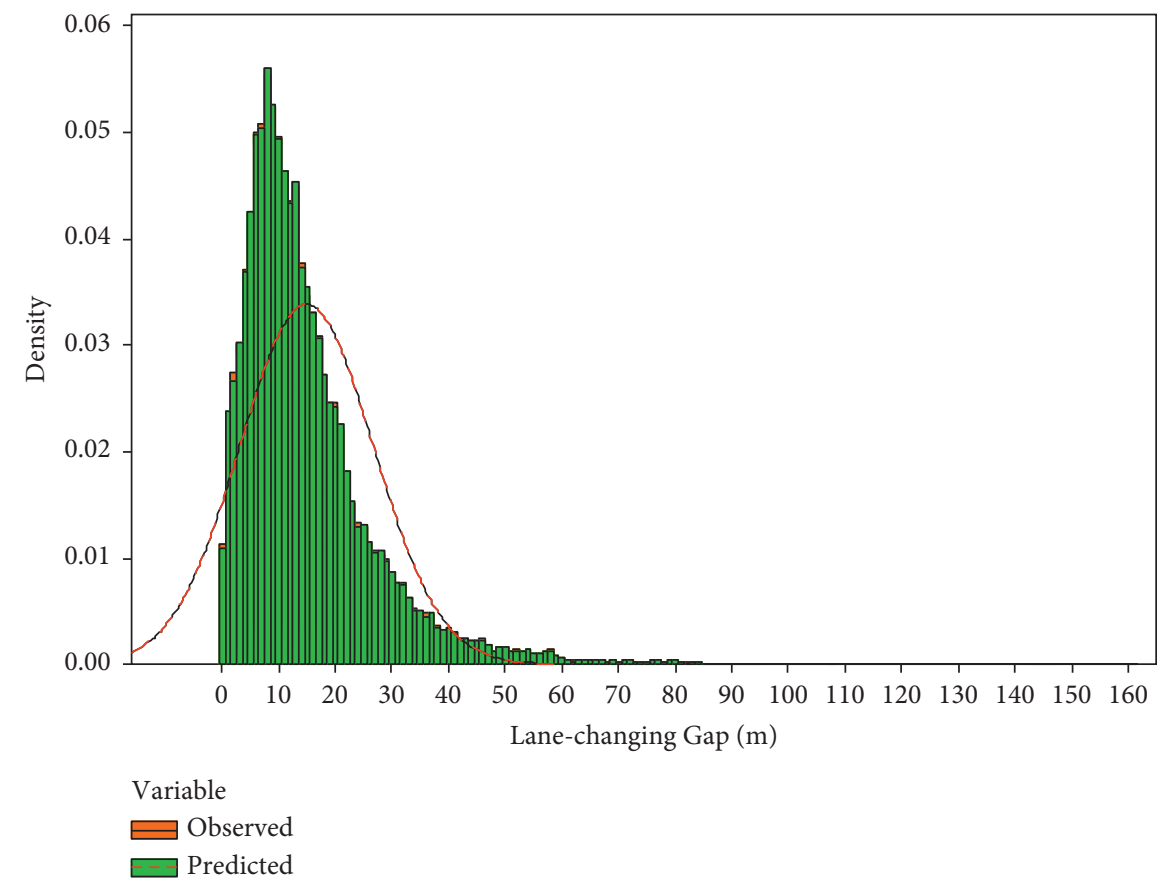

FIGURE 14: Gap distribution of LCs with NN model.

\subsection{Comparison of SVR- and NN-Based LC Models}

6.4.1. Supporting Vector Regression. Researchers have successfully developed and employed machine learning-based $\mathrm{CF}$ models in recent years, attempting to learn CF maneuvers through a massive amount of human driving $\mathrm{CF}$ data $[44,78]$. Machine learning techniques may derive the $\mathrm{CF}$ behavior of drivers and capture the possible connections between different variables that could affect CF behavior. The present study adopted a machine learning framework for LC maneuver analysis and comparison to the introduced NN-based model. Concerning machine learning approaches, SVMs have been increasingly attractive in light of their high predictive performance. Several studies demonstrated SVMs to yield more satisfactory outcomes as compared to the results of statistical and other machine learning techniques $[79,80]$. One can divide SVMs into classification SVMs and SVR machines. The former is employed for classification problems, while the latter is utilized for predicting continuous variables. SVR has proper generalizability and can deal with nonlinear problems. It has been successfully used for several real-life problems. SVR performs regularization error minimization and empirical risk minimization simultaneously with a proper penalty factor [81-83]. Consequently, the present work adopted SVR for LC trajectory prediction.

The two-dimensional lateral and longitudinal positions of each vehicle concerning the following and leading vehicles on the present and target lanes were derived from the NGSIM data. The SVR model inputs included the time sequence historical position data of the subject and four adjacent vehicles, as shown in Figure 2. The same training 
and testing datasets were applied to the NN and SVR models. Similar to the NN model, the two-dimensional subject vehicle positions were predicted in the following time steps. Let $\left[\left(X_{i}, y_{i}\right), \ldots,\left(X_{n}, y_{n}\right)\right]$, in which $X_{i}$ is the input and $y_{i}$ is the target, be the training dataset. The objective of $\varepsilon$-SVR is determining function $f(X)$ for which (a) has a maximum deviation of $\varepsilon$ from the target, and (b) has the highest possible flatness [84]. Particularly, the present study applied $\mathcal{\varepsilon}$-SVR with radial basis functions:

$$
f(X, W)=\sum_{j=1}^{n} W_{j} \exp \left(-\gamma\left\|X-X_{j}\right\|^{2}\right) .
$$

$\gamma$ is a variable, and vectors $X_{j}$ are inputs from the training dataset. To minimize the function, it is required to determine the unknown parameter vector $\mathrm{W}$ :

$$
\operatorname{Min} \frac{1}{2}\|W\|+C \sum_{i=1}^{n}\left(\operatorname{Max}\left|y_{i}-f\left(X_{i}, W\right)\right|-\varepsilon, 0\right),
$$

in which $C>0$ controls the trade-off between the flatness of $f(0)$ and the quantity up to which deviations above $\varepsilon$ are tolerated. Convex programming methods were employed to solve the dual of this optimization problem [84]. The normalization of the preprocessed data was carried out before training and testing (5). Then, the parameters $\varepsilon, \gamma$, and C were incorporated into the $\varepsilon$-SVR. The objective was to find values for these parameters for the predictive power maximization of the models. Eventually, the process was performed by combining grid-search and cross-validation methods [83].

The SVR model was trained and tested using a personal computer with a $1.78 \mathrm{GHz} \mathrm{CPU}$. To obtain optimal performance, SVR required a computational time of $4.0 \mathrm{~s}$. The training and testing of SVR lasted for $540 \mathrm{~s}$.

6.4.2. Comparison Results. As sufficient information on $\mathrm{NN}$ models was unavailable, we chose to adopt an NN-based LC model. In fact, NN success was a motivation of the work. Furthermore, CF approaches have recently been developed and applied based on machine learning. However, LC modeling has not been considered as frequently as $\mathrm{CF}$ modeling. To compare the proposed LC model, we adopted an SVR approach (machine learning) in order to analyze LC maneuvers. It was a challenging task to apply the SVR model with the same inputs as those of the NN. However, they both properly predicted trajectories with sufficient accuracy, capturing the longitudinal and lateral motions of the vehicles. Other models could be used for lane changing, but a review of data-driven $\mathrm{LC}$ researches indicates that NNs have been the most exciting instruments. Despite the development of some LCI and LCD-based NN approaches, a large number of questions remain yet to be answered, so that LC behavior could be understood. For example, long short-term memory (LSTM) could be a choice. According to the model inputs, which are spatial information in two dimensions (the lateral and longitudinal positions of the subject and four surrounding vehicles), we decided to use NN for LC prediction. As mentioned, $\mathrm{NN}$ success was a motivation of the authors. In fact, the use of the longitudinal and lateral vehicle positions as the NN inputs is the innovation of our work. Furthermore, LSTM takes more longer to train than NN, and LSTM is easy to overfit. Thus, the SVR and NN approaches can be said to have good performance. The NNbased and SVR-based LC models were compared. The same number of trajectories was employed for making comparisons. Also, the same set of data was utilized to train and test the SVR model in the form of an NN model. $15 \%$ of the data (i.e., sixty samples) were exploited in the testing phase, while $70 \%$ and $15 \%$ of the data were used for training and validation, respectively. Table 5 provides the MSE results of the two models. According to Table 5, the NN and SVR models yielded very close results.

Numerical tests were performed using the SVR model for the trajectory prediction of the sixty testing vehicles. MSE was utilized as an index of performance throughout the LC process. Figure 15 depicts the LC trajectory predictions of the testing vehicles (from the testing dataset).

Figure 16 shows the MSE values of the SVR and NN models for five vehicles. Also, Figure 17 demonstrates the trajectory observations and predictions of the same vehicles. As can be seen, the two models' trajectory predictions agree well with the observations and are capable of capturing lateral and longitudinal vehicle movements and SVR shows better performance than NN model. This, in turn, proves that the SVR and NN models have desirable performance. Furthermore, the results show that the SVR model is significantly similar to the $\mathrm{NN}$ model in trajectory prediction. A comparison of these models may demonstrate their ability to cope with overfitting.

Additionally, the model's predictive capability in reproducing macroscopic patterns can result from this study. The introduced models could make precise LC predictions of vehicles under small trajectory errors and satisfactory accuracy. In fact, NN and SVR models predict LC trajectories at time steps with the positions of both the subject and adjacent vehicles as the model input. After that, the effects of LC trajectory prediction can be seen in macroscopic patterns. For instance, the effect of LC trajectories on traffic flow at each time step predicted by proposed models can be determined and compared with the real data.

\subsubsection{Comparison of Proposed Models with New Input} Variables. The main innovative contribution is the parameters chosen for the model inputs. Finally, the proposed models were compared under various variables. To estimate LC behavior, it is required to select variables that could be measured using in-vehicle sensors. At the same time, it is important that multicollinearity is avoided by the traffic variables. For instance, since they have strong correlations, individual vehicle speeds and speed differences between different vehicles cannot be selected at the same time. Likewise, the vehicle type and gap are substantially intercorrelated and should not be simultaneously incorporated into the model. Eventually, a total of 11 variables were incorporated, as reported in Table 6. 
TABLE 5: MSE results of NN and SVR models.

\begin{tabular}{lr}
\hline Model & MSE \\
\hline NN & 0.023943 \\
SVR & 0.022643 \\
\hline
\end{tabular}

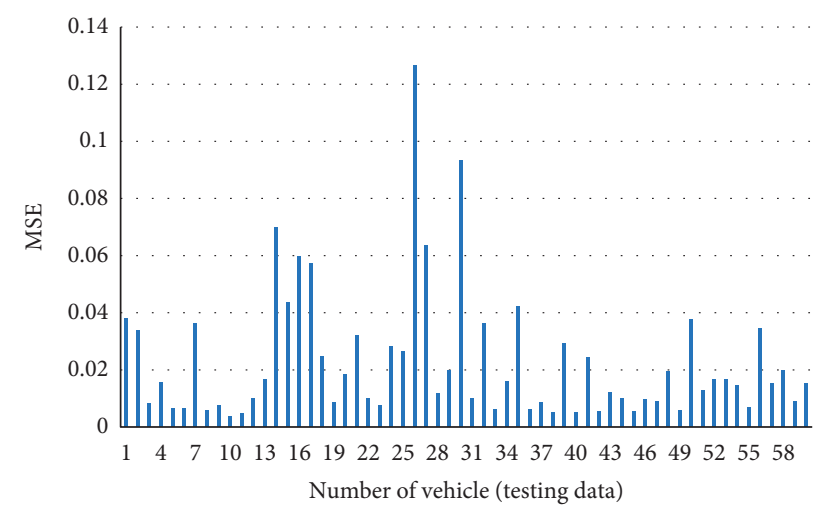

- MSE_testing data (SVR model)

FIGURE 15: LC trajectory predictions of the testing vehicles of the SVR model.

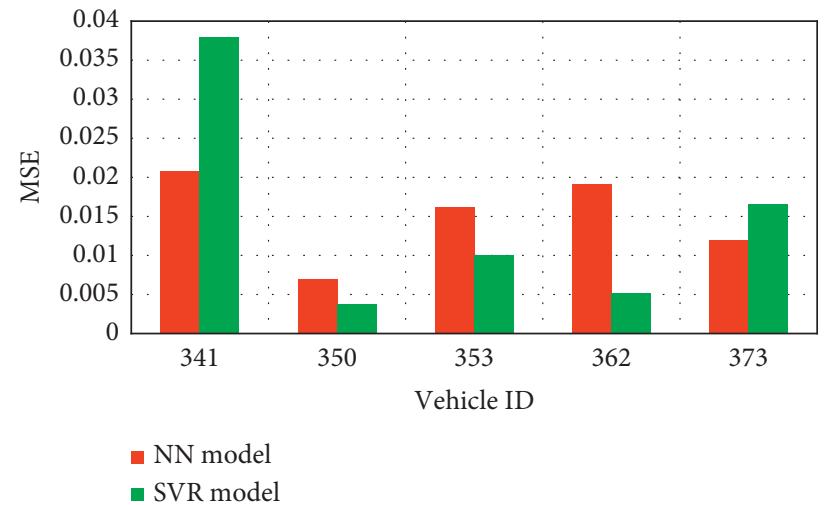

FIGURE 16: MSE of the predicted LC trajectories in NN and SVR models for 5 same vehicles.

The MSE results of both the proposed models and the new ones with different variables are shown in Table 7. As can be seen, the proposed models yielded a lower MSE than the ones with new inputs, and there is significant difference between models with position and new inputs.

Table 8 shows the MSE values of the SVR and NN models for five vehicles under both the position inputs and new inputs. As can be seen, the two models' trajectory predictions agree well with the observations and are capable of capturing lateral and longitudinal vehicle movements. This, in turn, proves that the SVR and NN models have desirable performance with position inputs. For instance, the observed and predicted trajectories of a vehicle under both the position inputs and new inputs are plotted in Figure 18. As can be seen, the trajectory predictions of the proposed models have greater consistency than the one with new inputs with the observed trajectories. Hence, the proposed models have higher performance than the new models. It can be said that information extraction from the lateral and longitudinal positions of the adjacent vehicles would yield 

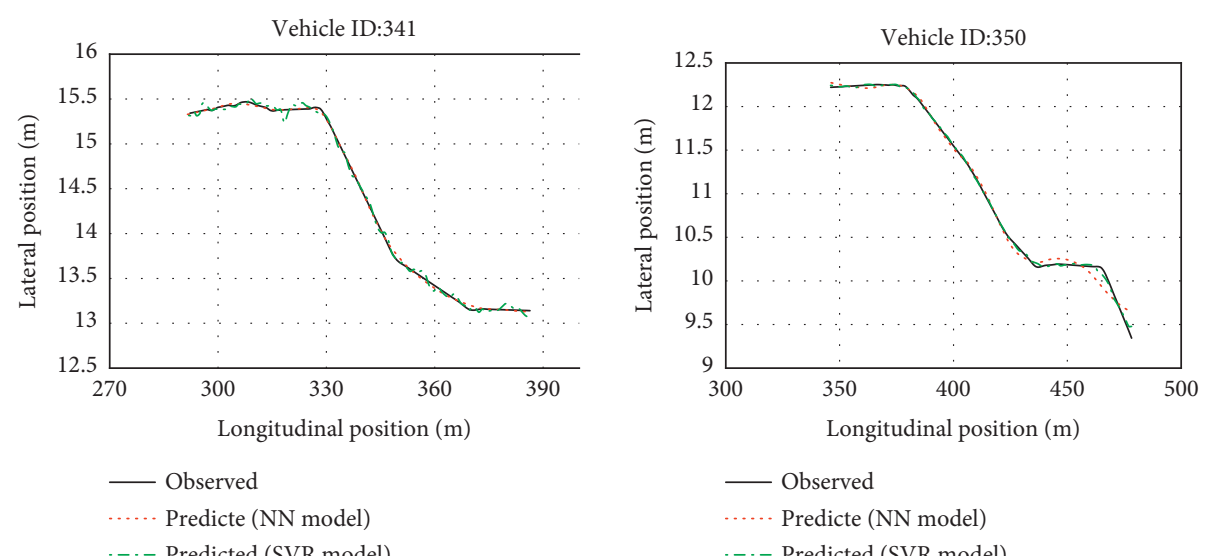

- Observed

-. Predicte (NN model)

-..- Predicted (SVR model)
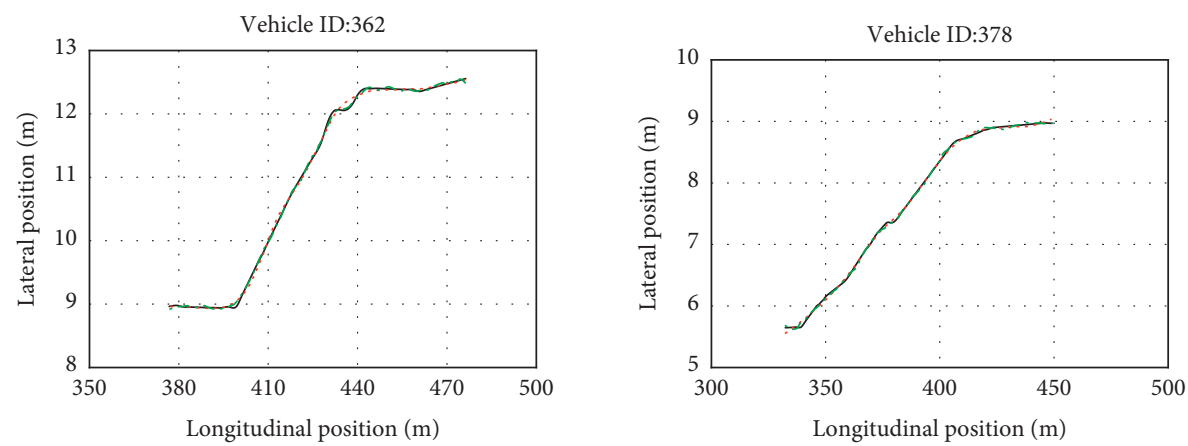

- Observed

..... Predicte (NN model)

...- Predicted (SVR model)

- Observed

...... Predicte (NN model)

...- Predicted (SVR model)

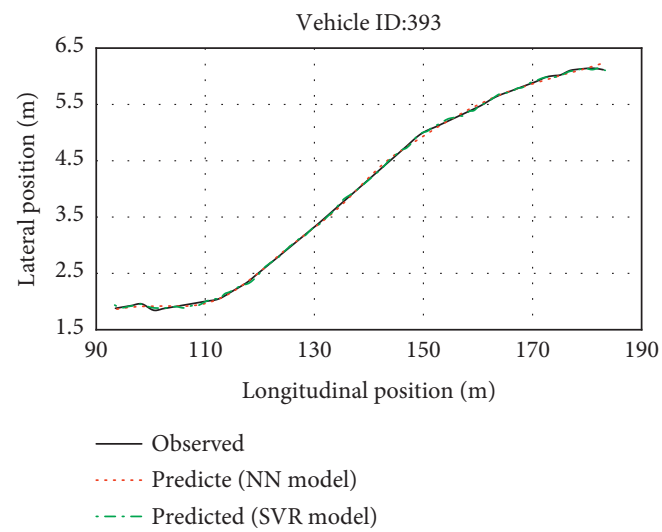

FIGURE 17: LC trajectories comparison between the SVR- and NN-based LC models. 
TABLE 6: New input variables of the NN and SVR models.

\begin{tabular}{lc}
\hline & Variables \\
\hline & $V(t)$ \\
$d 1(t)$ & $d 2(t)$ \\
& $d 3(t)$ \\
& $d 4(t)$ \\
Inputs & $V 1(t)$ \\
& $V 2(t)$ \\
& $V 3(t)$ \\
& $V 4(t)$ \\
\hline \multirow{2}{*}{ Outputs } & $X(t+\Delta t)$ : lateral position of the subject vehicle at next time step \\
& $Y(t+\Delta t)$ : longitudinal position of the subject vehicle at next time step \\
\hline
\end{tabular}

TABLE 7: MSE under different variables.

\begin{tabular}{lcc}
\hline NN and SVR models input & MSE (NN model) & MSE (SVR model) \\
\hline Position variables & 0.023943 & 0.022643 \\
New variables & 2.42 & 1.26 \\
\hline
\end{tabular}

TABLE 8: MSE of the predicted LC trajectories in NN and SVR models for 5 same vehicles under both the position inputs and new inputs.

\begin{tabular}{lcccc}
\hline Vehicle ID & NN model-position inputs & SVR model-position inputs & NN model-new inputs & SVR model-new inputs \\
\hline 341 & 0.0207 & 0.0379 & 1.807 & 0.32 \\
350 & 0.00699 & 0.0037 & 0.403 & 0.86 \\
362 & 0.0162 & 0.01 & 0.372 & 2.91 \\
378 & 0.0191 & 0.0052 & 1.011 & 1.532 \\
393 & 0.0119 & 0.0165 & 0.185 & 2.468 \\
\hline
\end{tabular}

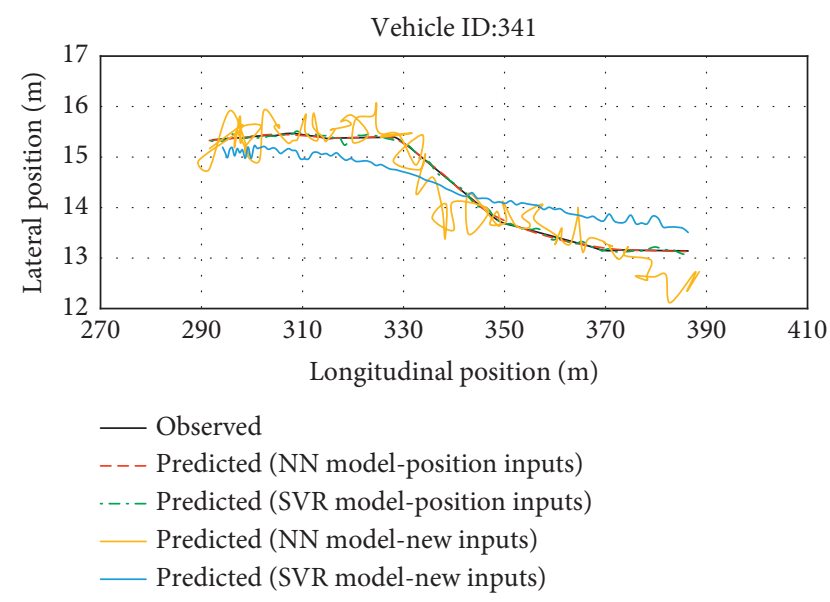

FIGURE 18: A comparison of the observed and predicted trajectories under position inputs and new inputs.

much better outcomes than using new inputs for the prediction of the subject vehicle position by the NN and SVR models.

\section{Conclusion and Future Works}

Neural networks (NNs) are employed primarily in light of their learning capability and incorporating uncertainties of real-life driving data. After learning from driving behavior data, an NN can produce vehicle states for reproducing driving styles. The present work developed an NN model for LC behavior modeling. The NGSIM database was employed to extract accurate LC records of two freeway sections, i.e., I-80 and US-101, in the United States. The developed model requires the position data of the adjacent vehicles solely to make vehicle trajectory predictions. In particular, the NN model incorporates the historical data right before LC implementation. The most important results of the present study include the following:

(i) The modeled results of the empirical data of vehicle trajectories revealed that the developed NN LC model could make accurate LC predictions of vehicles.

(ii) The experimental results suggested that the proposed technique could make accurate LC beginning/end estimates, demonstrating the beginning/ 
end predictions corresponding to feasible LC behavior.

(iii) The performance evaluation of the NN model was performed through several trajectory data points of LC and adjacent vehicles, i.e., the following and leading vehicles, on the present and target lanes. The proposed model was found to yield proper LC trajectory estimates of all vehicles concerning LC speed observation fluctuations.

(iv) For the characteristic validation of LC behavior, the comparison of the NN-reproduced LC gap distributions to the real-life data was carried out. The characteristics of the LC gap distributions showed no statistically significant difference from real-life LC behavior.

(v) It was found that the SVR model was significantly similar to the NN model in LC trajectory prediction. A comparison of the two models demonstrated their capability of coping with overfitting.

(vi) The MSE results of both proposed models and the new ones with different variables have shown that the proposed models yielded a lower MSE than the ones with new inputs, and there is a significant difference between models with position and new inputs.

As future works, state-of-the-art models like game theory models would be considered for lane-changing modeling. Although the game theory approach has been used for modeling lane-changing decisions in the literature [1], lane-changing trajectory prediction can be tested with the game theory approach.

Also, the proposed model would be integrated in numerical simulations like AIMSUN. To integrate NN model in numerical simulations like AIMSUN, the output of $\mathrm{NN}$ model is used for calculating two local parameters (distance zones 1 and 2 [85]) that have the greatest influence on lane changing in AIMSUN. Then, these two local parameters are used in AIMSUN, and the average flows and speeds of all vehicles on the section are predicted by simulation model.

\section{Data Availability}

The original NGSIM data is open to download at https://ops. fhwa.dot.gov/trafficanalysistools/ngsim.htm.

\section{Conflicts of Interest}

The authors declare that there are no conflicts of interest regarding the publication of this paper.

\section{References}

[1] Y. Ali, Z. Zheng, M. M. Haque, and M. Wang, "A game theory-based approach for modelling mandatory lanechanging behaviour in a connected environment," Transportation Research Part C: Emerging Technologies, vol. 106, pp. 220-242, 2019.
[2] Y. Ali, Z. Zheng, M. M. Haque, M. Yildirimoglu, and S. Washington, "Detecting, analysing, and modelling failed lane-changing attempts in traditional and connected environments," Analytic Methods in Accident Research, vol. 28, Article ID 100138, 2020.

[3] D. Ngoduy, "Analytical studies on the instabilities of heterogeneous intelligent traffic flow," Communications in Nonlinear Science and Numerical Simulation, vol. 18, no. 10, pp. 2699-2706, 2013.

[4] C. Colombaroni and G. Fusco, "Artificial neural network models for car following: experimental analysis and calibration issues," Journal of Intelligent Transportation Systems, vol. 18, no. 1, pp. 5-16, 2014.

[5] D. Ngoduy, "Effect of the car-following combinations on the instability of heterogeneous traffic flow," Transportation Business: Transport Dynamics, vol. 3, no. 1, pp. 44-58, 2015.

[6] L. Li and X. Chen, "Vehicle headway modeling and its inferences in macroscopic/microscopic traffic flow theory: a survey," Transportation Research Part C: Emerging Technologies, vol. 76, pp. 170-188, 2017.

[7] Z. Zheng, S. Ahn, D. Chen, and J. Laval, "Freeway traffic oscillations: microscopic analysis of formations and propagations using wavelet transform," Procedia - Social and Behavioral Sciences, vol. 17, pp. 702-716, 2011.

[8] D. Chen, J. Laval, Z. Zheng, and S. Ahn, "A behavioral carfollowing model that captures traffic oscillations," Transportation Research Part B: Methodological, vol. 46, no. 6, pp. 744-761, 2012.

[9] D. Chen, J. A. Laval, S. Ahn, and Z. Zheng, "Microscopic traffic hysteresis in traffic oscillations: a behavioral perspective," Transportation Research Part B: Methodological, vol. 46, no. 10, pp. 1440-1453, 2012.

[10] Y. Ali, Z. Zheng, M. M. Haque, M. Yildirimoglu, and S. Washington, "CLACD: a complete LAne-Changing decision modeling framework for the connected and traditional environments," Transportation Research Part C: Emerging Technologies, vol. 128, Article ID 103162, 2021.

[11] T. F. Golob, W. W. Recker, and V. M. Alvarez, "Safety aspects of freeway weaving sections," Transportation Research Part A: Policy and Practice, vol. 38, no. 1, pp. 35-51, 2004.

[12] J. Lee and M. J. Cassidy, "An empirical and theoretical study of freeway weave bottlenecks," 2008, https://escholarship.org/ uc/item/2970816w.

[13] A. Srivastava and N. Geroliminis, "Empirical observations of capacity drop in freeway merges with ramp control and integration in a first-order model," Transportation Research Part C: Emerging Technologies, vol. 30, pp. 161-177, 2013.

[14] Y. Ali, M. M. Haque, and Z. Zheng, "An Extreme Value Theory approach to estimate crash risk during mandatory lane-changing in a connected environment," Analytic Methods in Accident Research, vol. 33, Article ID 100193, 2022.

[15] S. Ahn and M. J. Cassidy, "Freeway traffic oscillations and vehicle lane-change maneuvers," in Transportation and Traffic Theory 2007. Papers Selected for Presentation at ISTTT17Engineering and Physical Sciences Research Council (Great Britain) Rees Jeffreys Road FundTransport Research FoundationTMS ConsultancyOve Arup and PartnersHong KongTransportation Planning (International) PTV AG, Amsterdam, 2007, http://worldcat.org/isbn/9780080453750.

[16] C. Chao Wang and B. Coifman, "The effect of lane-change maneuvers on a simplified car-following theory," IEEE Transactions on Intelligent Transportation Systems, vol. 9, no. 3, pp. 523-535, 2008. 
[17] M. J. Cassidy and J. Rudjanakanoknad, "Increasing the capacity of an isolated merge by metering its on-ramp," Transportation Research Part B: Methodological, vol. 39, no. 10, pp. 896-913, 2005.

[18] D. Chen and S. Ahn, "Capacity-drop at extended bottlenecks: merge, diverge, and weave," Transportation Research Part B: Methodological, vol. 108, pp. 1-20, 2018.

[19] J. Laval, M. Cassidy, and C. Daganzo, "Impacts of lane changes at merge bottlenecks: a theory and strategies to maximize capacity," in Traffic and Granular Flow'05, pp. 577-586, Springer, Switzerland AG, 2007.

[20] B. Ran, Y. Cheng, S. Li et al., "Connected automated vehicle highway systems and methods," Google Patents, 2019, https://patentimages.storage.googleapis.com/7e/19/46/ 1d647d09326de3/US10380886.pdf.

[21] A. Kesting, M. Treiber, and D. Helbing, "General lanechanging model MOBIL for car-following models," Transportation Research Record: Journal of the Transportation Research Board, vol. 1999, no. 1, pp. 86-94, 2007.

[22] F. Altché and A. de La Fortelle, "An LSTM network for highway trajectory prediction," in Proceedings of the 2017 IEEE 20th International Conference on Intelligent Transportation Systems (ITSC), pp. 353-359, IEEE, Yokohama, Japan, October 2017.

[23] A. Zyner, S. Worrall, J. Ward, and E. Nebot, "Long short term memory for driver intent prediction," in Proceedings of the 2017 IEEE Intelligent Vehicles Symposium (IV), pp. 1484-1489, IEEE, Los Angeles, CA, USA, June 2017.

[24] L. Waymo, "On the road to fully self-driving," pp. 1-43, Waymo Safety Report, 2017, https://www.auto-mat.ch/ wAssets/docs/171019_waymo-safety-report-2017-10.pdf.

[25] Y. Ali, M. C. Bliemer, Z. Zheng, and M. M. Haque, "Cooperate or not? Exploring drivers' interactions and response times to a lane-changing request in a connected environment," Transportation Research Part C: Emerging Technologies, vol. 120, Article ID 102816, 2020.

[26] A. Cosgun, L. Ma, J. Chiu et al., “Towards full automated drive in urban environments: a demonstration in gomentum station, California," in Proceedings of the 2017 IEEE Intelligent Vehicles Symposium (IV), pp. 1811-1818, IEEE, Los Angeles, CA, USA, June 2017.

[27] J. Li, B. Dai, X. Li, C. Li, and Y. Di, "A real-time and predictive trajectory-generation motion planner for autonomous ground vehicles," in Proceedings of the 2017 9th International Conference on Intelligent Human-Machine Systems and Cybernetics (IHMSC), pp. 108-113, IEEE, Hangzhou, China, August 2017.

[28] H. Woo, Y. Ji, H. Kono et al., "Lane-change detection based on vehicle-trajectory prediction," IEEE Robotics and Automation Letters, vol. 2, no. 2, pp. 1109-1116, 2017.

[29] R. Zhang, L. Cao, S. Bao, and J. Tan, "A method for connected vehicle trajectory prediction and collision warning algorithm based on V2V communication," International Journal of Crashworthiness, vol. 22, no. 1, pp. 15-25, 2017.

[30] N. Djuric, V. Radosavljevic, H. Cui et al., "Short-term motion prediction of traffic actors for autonomous driving using deep convolutional networks," 2018, https://www.arxiv-vanity. com/papers/1808.05819/.

[31] C. Ding, W. Wang, X. Wang, and M. Baumann, "A neural network model for driver's lane-changing trajectory prediction in urban traffic flow," Mathematical Problems in Engineering, vol. 2013, 2013.

[32] T. Shamir, "How should an autonomous vehicle overtake a slower moving vehicle: design and analysis of an optimal trajectory," IEEE Transactions on Automatic Control, vol. 49, no. 4, pp. 607-610, 2004.

[33] W. Yao, H. Zhao, P. Bonnifait, and H. Zha, "Lane change trajectory prediction by using recorded human driving data," in Proceedings of the 2013 IEEE Intelligent Vehicles Symposium (IV), pp. 430-436, IEEE, Gold Coast, QLD, Australia, June 2013.

[34] Z. Zhao, W. Chen, X. Wu, P. C. Y. Chen, and J. Liu, "LSTM network: a deep learning approach for short-term traffic forecast," IET Intelligent Transport Systems, vol. 11, no. 2, pp. 68-75, 2017.

[35] Y. Dou, F. Yan, and D. Feng, "Lane changing prediction at highway lane drops using support vector machine and artificial neural network classifiers," in Proceedings of the 2016 IEEE International Conference on Advanced Intelligent Mechatronics (AIM), pp. 901-906, IEEE, Banff, AB, Canada, July 2016.

[36] J. Gao, Y. L. Murphey, and H. Zhu, "Multivariate time series prediction of lane changing behavior using deep neural network," Applied Intelligence, vol. 48, no. 10, pp. 3523-3537, 2018.

[37] P. Kumar, M. Perrollaz, S. Lefevre, and C. Laugier, "Learningbased approach for online lane change intention prediction," in Proceedings of the 2013 IEEE Intelligent Vehicles Symposium (IV), pp. 797-802, IEEE, Gold Coast, QLD, Australia, June 2013.

[38] K. Li, X. Wang, Y. Xu, and J. Wang, "Lane changing intention recognition based on speech recognition models," Transportation Research Part C: Emerging Technologies, vol. 69, pp. 497-514, 2016.

[39] L. Li, M. Zhang, and R. Liu, "The application of Bayesian filter and neural networks in lane changing prediction," in Proceedings of the 5th International Conference on Civil Engineering and Transportation, Seoul, Korea, July 2015.

[40] X. Zhang, J. Sun, X. Qi, and J. Sun, "Simultaneous modeling of car-following and lane-changing behaviors using deep learning," Transportation Research Part C: Emerging Technologies, vol. 104, pp. 287-304, 2019.

[41] E. C. B. Olsen, "Modeling slow lead vehicle lane changing," Virginia Tech, 2003, https://www.researchgate.net/ publication/279681796_Modeling_Slow_Lead_Vehicle_ Lane_Changing.

[42] V. L. Knoop, S. P. Hoogendoorn, Y. Shiomi, and C. Buisson, "Quantifying the number of lane changes in traffic," Transportation Research Record: Journal of the Transportation Research Board, vol. 2278, no. 1, pp. 31-41, 2012.

[43] J. Chovan, L. Tijerina, G. Alexander, and D. Hendricks, "Examination of lane change crashes and potential IVHS countermeasures," 1994, https://ntlrepository.blob.core. windows.net/lib/16000/16600/16677/PB2000104485.pdf.

[44] B. Zhou, Y. Wang, G. Yu, and X. Wu, "A lane-change trajectory model from drivers' vision view," Transportation Research Part C: Emerging Technologies, vol. 85, pp. 609-627, 2017.

[45] Y. Wang, D. Zhang, Y. Liu, B. Dai, and L. H. Lee, "Enhancing transportation systems via deep learning: a survey," Transportation Research Part C: Emerging Technologies, vol. 99, pp. 144-163, 2019.

[46] J. Zheng, K. Suzuki, and M. Fujita, "Predicting driver's lanechanging decisions using a neural network model," Simulation Modelling Practice and Theory, vol. 42, pp. 73-83, 2014.

[47] J. G. Hunt and G. D. Lyons, "Modelling dual carriageway lane changing using neural networks," Transportation Research Part C: Emerging Technologies, vol. 2, no. 4, pp. 231-245, 1994. 
[48] L. Li, M. Zhang, and R. Liu, "The application of Bayesian filter and neural networks in lane changing prediction," in Proceedings of the 5th International Conference on Civil Engineering and Transportation, pp. 2004-2007, Seoul, Korea, July 2015.

[49] M. Ke and H. Wang, "Lane-changing decision model for connected and automated vehicle based on back-propagation neural network," in Proceedings of the International Conference on Transportation and Development 2020, pp. 163-173, American Society of Civil Engineers, Reston, VA, November 2020.

[50] Z. Zheng, "Recent developments and research needs in modeling lane changing," Transportation Research Part B: Methodological, vol. 60, pp. 16-32, 2014.

[51] D. Yang, S. Zheng, C. Wen, P. J. Jin, and B. Ran, "A dynamic lane-changing trajectory planning model for automated vehicles," Transportation Research Part C: Emerging Technologies, vol. 95, pp. 228-247, 2018.

[52] H. Yu, H. E. Tseng, and R. Langari, "A human-like game theory-based controller for automatic lane changing," Transportation Research Part C: Emerging Technologies, vol. 88, pp. 140-158, 2018.

[53] T. Awal, M. Murshed, and M. Ali, "An efficient cooperative lane-changing algorithm for sensor-and communicationenabled automated vehicles," in Proceedings of the 2015 IEEE Intelligent Vehicles Symposium (IV), pp. 1328-1333, IEEE, Seoul, Korea, July 2015.

[54] V. Mahajan, C. Katrakazas, and C. Antoniou, "Prediction of lane-changing maneuvers with automatic labeling and deep learning," Transportation Research Record: Journal of the Transportation Research Board, vol. 2674, no. 7, pp. 336-347, Article ID 0361198120922210, 2020.

[55] D.-F. Xie, Z.-Z. Fang, B. Jia, and Z. He, "A data-driven lanechanging model based on deep learning," Transportation Research Part C: Emerging Technologies, vol. 106, pp. 41-60, 2019.

[56] S. Lee, D. Ngoduy, and M. Keyvan-Ekbatani, "Integrated deep learning and stochastic car-following model for traffic dynamics on multi-lane freeways," Transportation Research Part C: Emerging Technologies, vol. 106, pp. 360-377, 2019.

[57] R. S. Tomar, S. Verma, and G. S. Tomar, "Prediction of lane change trajectories through neural network," in Proceedings of the 2010 International Conference on Computational Intelligence and Communication Networks, IEEE, pp. 249-253, Bhopal, India, November 2010.

[58] J. Tang, F. Liu, W. Zhang, R. Ke, and Y. Zou, "Lane-changes prediction based on adaptive fuzzy neural network," Expert Systems with Applications, vol. 91, pp. 452-463, 2018.

[59] J. Tang, S. Yu, F. Liu, X. Chen, and H. Huang, "A hierarchical prediction model for lane-changes based on combination of fuzzy C-means and adaptive neural network," Expert Systems with Applications, vol. 130, pp. 265-275, 2019.

[60] J. Peng, Y. Guo, R. Fu, W. Yuan, and C. Wang, "Multi-parameter prediction of drivers' lane-changing behaviour with neural network model," Applied Ergonomics, vol. 50, pp. 207-217, 2015.

[61] C. F. Choudhury and M. E. Ben-Akiva, "Modelling driving decisions: a latent plan approach," Transportmetrica: Transportation Science, vol. 9, no. 6, pp. 546-566, 2013.

[62] Z. He, Y. Lv, L. Lu, and W. Guan, "Constructing spatiotemporal speed contour diagrams: using rectangular or nonrectangular parallelogram cells?" Transportation Business: Transport Dynamics, vol. 7, no. 1, pp. 44-60, 2019.
[63] Z. He, W. Zhang, and N. Jia, "Estimating carbon dioxide emissions of freeway traffic: a spatiotemporal cell-based model," IEEE Transactions on Intelligent Transportation Systems, vol. 21, pp. 1976-1986, 2019.

[64] Z. He, L. Zheng, and W. Guan, "A simple nonparametric carfollowing model driven by field data," Transportation Research Part B: Methodological, vol. 80, pp. 185-201, 2015.

[65] C. Thiemann, M. Treiber, and A. Kesting, "Estimating acceleration and lane-changing dynamics from next generation simulation trajectory data," Transportation Research Record: Journal of the Transportation Research Board, vol. 2088, no. 1, pp. 90-101, 2008.

[66] X. Wang, R. Jiang, L. Li, Y. Lin, X. Zheng, and F.-Y. Wang, "Capturing car-following behaviors by deep learning," IEEE Transactions on Intelligent Transportation Systems, vol. 19, pp. 910-920, 2017.

[67] J. Zheng, K. Suzuki, and M. Fujita, "Car-following behavior with instantaneous driver-vehicle reaction delay: a neuralnetwork-based methodology," Transportation Research Part C: Emerging Technologies, vol. 36, pp. 339-351, 2013.

[68] Z. Zheng, S. Ahn, D. Chen, and J. Laval, "The effects of lanechanging on the immediate follower: anticipation, relaxation, and change in driver characteristics," Transportation Research Part C: Emerging Technologies, vol. 26, pp. 367-379, 2013.

[69] M. Li, Z. Li, C. Xu, and T. Liu, "Short-term prediction of safety and operation impacts of lane changes in oscillations with empirical vehicle trajectories," Accident Analysis \& Prevention, vol. 135, p. 105345, Article ID 105345, 2020.

[70] Y. Ali, M. C. J. Bliemer, Z. Zheng, and M. M. Haque, "Comparing the usefulness of real-time driving aids in a connected environment during mandatory and discretionary lane-changing manoeuvres," Transportation Research Part C: Emerging Technologies, vol. 121, Article ID 102871, 2020.

[71] J. Sun, J. Ouyang, and J. Yang, "Modeling and analysis of merging behavior at expressway on-ramp bottlenecks," Transportation Research Record: Journal of the Transportation Research Board, vol. 2421, no. 1, pp. 74-81, 2014.

[72] T. Kohonen, "An introduction to neural computing," Neural Networks, vol. 1, no. 1, pp. 3-16, 1988.

[73] P. S. Gowrishankar and P. S. Satyanarayana, "Neural network based traffic prediction for wireless data networks," International Journal of Computational Intelligence Systems, vol. 1, no. 4, pp. 379-389, 2008.

[74] E. G. Tsionas, P. G. Michaelides, and A. T. Vouldis, "Global approximations to cost and production functions using artificial neural networks," International Journal of Computational Intelligence Systems, vol. 2, no. 2, pp. 132-139, 2009.

[75] E. Zio, "Neural networks simulation of the transport of contaminants in groundwater," International Journal of Computational Intelligence Systems, vol. 2, no. 3, pp. 267-276, 2009.

[76] C. Yin, L. Rosendahl, and Z. Luo, "Methods to improve prediction performance of ANN models," Simulation Modelling Practice and Theory, vol. 11, no. 3-4, pp. 211-222, 2003.

[77] L. Li, C. Lv, D. Cao, and J. Zhang, "Retrieving common discretionary lane changing characteristics from trajectories," IEEE Transactions on Vehicular Technology, vol. 67, pp. 2014-2024, 2017.

[78] D. Yang, L. Zhu, Y. Liu, D. Wu, and B. Ran, "A novel carfollowing control model combining machine learning and kinematics models for automated vehicles," IEEE Transactions on Intelligent Transportation Systems, vol. 20, pp. 1991-2000, 2018. 
[79] A. Iranitalab and A. Khattak, "Comparison of four statistical and machine learning methods for crash severity prediction," Accident Analysis \& Prevention, vol. 108, pp. 27-36, 2017.

[80] J. Zhang, Z. Li, Z. Pu, and C. Xu, "Comparing prediction performance for crash injury severity among various machine learning and statistical methods," IEEE Access, vol. 6, pp. 60079-60087, 2018.

[81] I. O. Alade, A. Bagudu, T. A. Oyehan, M. A. A. Rahman, T. A. Saleh, and S. O. Olatunji, "Estimating the refractive index of oxygenated and deoxygenated hemoglobin using genetic algorithm - support vector regression model," Computer Methods and Programs in Biomedicine, vol. 163, pp. 135-142, 2018.

[82] K. Cheng, Z. Lu, Y. Wei, Y. Shi, and Y. Zhou, "Mixed kernel function support vector regression for global sensitivity analysis," Mechanical Systems and Signal Processing, vol. 96, pp. 201-214, 2017.

[83] N. Marković, S. Milinković, K. S. Tikhonov, and P. Schonfeld, "Analyzing passenger train arrival delays with support vector regression," Transportation Research Part C: Emerging Technologies, vol. 56, pp. 251-262, 2015.

[84] A. J. Smola and B. Schölkopf, "A tutorial on support vector regression," Statistics and Computing, vol. 14, no. 3, pp. 199-222, 2004.

[85] P. Hidas, "Lane changing and merging under congested conditions in traffic simulation models," WIT Transactions on The Built Environment, vol. 77, 2005. 